University of Louisville

ThinkIR: The University of Louisville's Institutional Repository

$5-2011$

\title{
CFD modeling of center cones, vortex breakers and pressure relief slits in a wind speed accelerator.
}

Michael D. Lucas 1988-

University of Louisville

Follow this and additional works at: https://ir.library.louisville.edu/etd

\section{Recommended Citation}

Lucas, Michael D. 1988-, "CFD modeling of center cones, vortex breakers and pressure relief slits in a wind speed accelerator." (2011). Electronic Theses and Dissertations. Paper 861.

https://doi.org/10.18297/etd/861

This Master's Thesis is brought to you for free and open access by ThinkIR: The University of Louisville's Institutional Repository. It has been accepted for inclusion in Electronic Theses and Dissertations by an authorized administrator of ThinkIR: The University of Louisville's Institutional Repository. This title appears here courtesy of the author, who has retained all other copyrights. For more information, please contact thinkir@louisville.edu. 


\author{
By \\ Michael D. Lucas \\ B. S., University of Louisville, 2010

\begin{abstract}
A Thesis
Submitted to the Faculty of the

University of Louisville

J. B. School of Engineering

as Partial Fulfillment of the Requirements

for the Professional Degree
\end{abstract}

MASTER OF ENGINEERING

Department of Chemical Engineering

May, 2011 

Submitted by:

Michael Lucas

A Thesis Approved On

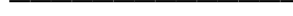

(Date)

by the Following Reading and Examination Committee:

R. Eric Berson, Thesis Director

Gerold Willing

Yongsheng Lian

William Komp 


\section{AKNOWLEDGEMENTS}

I would like to thank Dr. Eric Berson for his guidance, as well as Dr. William

Komp for his technical input. I would also like to thank David Russ and WEST Wind Power for their contributions and support. 


\section{ABSTRACT \\ CFD MODELING OF CENTER CONES, VORTEX BREAKERS AND PRESSURE RELIEF SLITS IN A WIND SPEED ACCELERATOR}

\section{Michael Lucas}

\section{March, 12, 2011}

Wind power has the potential to provide access to electricity to areas lacking the resources to create industrial power plants, as well as supplement residential energy supplies. The low resource investment and ease of installation make it ideal for these scenarios; however, it is limited by the availability of a stable power source. Only $13 \%$ of the world's land area experiences wind speeds high enough to be usable by current technology. To improve this percentage, the use of wind concentrators has been suggested. A turbine within a concentrator would experience a higher wind speed than the surrounding body of air, reducing the ambient wind speed requirement to generate electricity. In this thesis, several concentrator designs were tested. In this thesis, several designs of the following concentrator components were tested: a flow straightener, vortex breaker, and pressure relief slits.

Fluent 12.1, a computational fluid dynamics (CFD) program, was used to model air flow patterns through a prototype wind concentrator and optimize its performance. Through this method, it was determined that a concentrator with a trumpet shaped entrance and exit is effective at concentrating wind energy. A long, thin center cone was most effective at accelerating a wind stream, while vortex breakers were ineffective. Maximum velocities were obtained with the addition of pressure-relief slits in the inlet 
portion. With an ambient inlet air stream of $2 \mathrm{~m} / \mathrm{s}$, CFD results predicted the concentrator would accelerate the air velocity to $5.17 \mathrm{~m} / \mathrm{s}$. The concentrator also predicted similar accelerations at higher inlet velocities. This data was validated by results provided by WEST Wind Power Inc. Their prototype, built from the optimized model's blueprints, observed wind speeds in the device throat within $8 \%$ of the predicted values. 


\section{TABLE OF CONTENTS}

SECTION

PAGE

APPROVAL PAGE

$\begin{array}{ll}\text { AKNOWLEDGEMENTS } & \text { iii }\end{array}$

ABSTRACT iv

TABLE OF CONTENTS V v

NOMENCLATURE viii

LIST OF TABLES $\quad \mathrm{x}$

LIST OF FIGURES

I. INTRODUCTION 1

II. LITERATURE REVIEW 3

A. Computational Fluid Dynamics 3

B. Realizable k-€ Model 5

C. Wind Concentration and Low Velocity Turbines 9

$\begin{array}{ll}\text { III. PROCEDURES } & 20\end{array}$

A. Geometry and Mesh Generation 20

B. Problem Solving in Fluent 27

C. Viewing Results 33

IV. RESULTS AND DISCUSSION 36

V. CONCLUSIONS AND RECOMMENDATIONS 56

$\begin{array}{lr}\text { A. Conclusions } & 56\end{array}$

B. Recommendations 56 
REFERENCES

APPENDIX - A

60

CURRICULUM VITAE

64 


\section{NOMENCLATURE}

$$
\begin{array}{lll}
E & = & \text { sensible enthalpy factor }(\mathrm{J} / \mathrm{kg}) \\
F & = & \text { net force vector }\left(\mathrm{N} / \mathrm{m}^{3}\right) \\
g & = & \text { gravity vector }\left(\mathrm{m} / \mathrm{s}^{2}\right) \\
k & = & \text { turbulent kinetic energy }\left(\mathrm{J} / \mathrm{kg} \mathrm{or} \mathrm{m}^{2} / \mathrm{s}^{2}\right) \\
p & = & \text { pressure }\left(\mathrm{N} / \mathrm{m}^{2}\right) \\
t & = & \text { Time }(\mathrm{s}) \\
T & = & \text { Temperature }(\mathrm{K}) \\
v & = & \text { velocity vector }(\mathrm{m} / \mathrm{s}) \\
X & = & \text { distance variable }(\mathrm{m}) \\
\tau & = & \text { stress tensor }\left(\mathrm{N} / \mathrm{m}^{2}\right) \\
C_{l-3 \epsilon} & = & \text { tuning constants from Fluent } \\
C_{c} & = & \text { concavity constant } \\
C_{s} & = & \text { scaling constant } \\
G_{b} & = & \text { kinetic energy generation due to buoyancy }\left(\mathrm{W} / \mathrm{m}^{3}\right) \\
G_{k} & = & \text { kinetic energy generation due to velocity }\left(\mathrm{W} / \mathrm{m}^{3}\right) \\
\nabla & = & \text { gradient } \\
k_{e f f} & = & \text { thermal conductivity }\left[\mathrm{W} /\left(\mathrm{m}^{*} \mathrm{~K}\right)\right] \\
S_{h} & = & \text { net heat flow rate }\left(\mathrm{W} / \mathrm{m}^{3}\right) \\
S_{k} & =
\end{array}
$$




\begin{tabular}{|c|c|c|}
\hline$S_{m}$ & $=$ & net mass flow rate $\left[\mathrm{kg} /\left(\mathrm{s}^{*} \mathrm{~m}^{3}\right)\right]$ \\
\hline$S_{\epsilon}$ & $=$ & user defined source term $\left[\left(\mathrm{W} /\left(\mathrm{s}^{*} \mathrm{~m}^{3}\right)\right]\right.$ \\
\hline$u_{j}$ & $=$ & scalar component of velocity vector $(\mathrm{m} / \mathrm{s})$ \\
\hline$x j$ & $=$ & direction component of velocity vector \\
\hline$Y_{M}$ & $=$ & turbulent energy generation due to compressibility \\
\hline$\epsilon$ & $=$ & rate of turbulent dissipation $\left(\mathrm{m}^{2} / \mathrm{s}^{3}\right)$ \\
\hline$\mu$ & $=$ & Viscosity $\left(\mathrm{N}^{*} \mathrm{~s} / \mathrm{m}^{2}\right)$ \\
\hline$\mu_{t}$ & $=$ & turbulent viscosity $\left(\mathrm{N}^{*} \mathrm{~s} / \mathrm{m}^{2}\right)$ \\
\hline$\sigma_{k}$ & $=$ & turbulent prandlt number for $\mathrm{k}$ \\
\hline$\sigma_{\epsilon}$ & $=$ & turbulent prandlt number for $\epsilon$ \\
\hline$\nabla$ & $=$ & del operator \\
\hline$\rho$ & $=$ & Density $\left(\mathrm{kg} / \mathrm{m}^{3}\right)$ \\
\hline
\end{tabular}




\section{LIST OF TABLES}

TABLE

PAGE

I. THROAT VELOCITIES WITH VARYING GEOMETRIES 38

II. THROAT VELOCITIES WITH DIFFERING VARYING LENGTHS 41

III. THROAT VELOCITIES WITH VARYING CONE DIAMETERS 43

IV. WIND CONCENTRATOR PROFILE COORDINATES 60 


\section{LIST OF FIGURES}

FIGURE

PAGE

1. Various Vertical Axis Wind Turbine Designs 12

2. Ultimate Wind Turbine 13

3. Turbine with Shroud or Diffuser 16

4. Loth's Turbine 18

5. Explicit Coordinates 21

6. Surface of Revolution 23

$\begin{array}{ll}\text { 7. Part Creation } & 24\end{array}$

8. Mesh Nodes 25

9. Output File 26

10. Viscous Model 27

11. Material Name 28

12. Boundary Conditions $\quad 29$

13. Spatial Discretization 30

14. Solution Controls 31

15. Moniters 32

16. Initialization and Calculation 33

17. Graphis and Animations: Creating a Plane 34

18. Graphics and Animations: Defining a Plane 35

19. Original Turbine Concept from West Wind Power Inc. 36 
20. Curves Representing Inlet Shapes. The Outer Edge of the Cone Inlet is at $0.5 \mathrm{~m}$

21. Destructive Eddies in a Parabolic Curve. Vectors Collide in Front of the Throat

22. New Turbine Design with Cone

23. Velocity Contours for Cone Lengths of 20, 40, 60 and $100 \mathrm{~cm}$

24. Velocity Contours for Cone Diameters of 2, 8, 14 and $20 \mathrm{~cm}$

25. Turbulent Kinetic Energy Profile of Preferred Cone Geometry

26. Velocity Profile of Preferred Cone Geometry

27. Velocity Profiles of Slit and Vortex Breaker Designs

28. Turbulence Profiles of Slit and Vortex Breaker Designs

29. Vortex Breaker

30. Slit Designs

31. Control Design for Slit Optimization

32. Slit Designs 2

33. Experimental Data

34. Front View (All Units in mm)

35. Side View (All Units in mm)

36. Rear View (All Units in mm) 


\section{INTRODUCTION}

According to a Stanford study, the potential accessible energy available globally from wind power exceeds the global energy demand by a factor of five (Archer and Jacobson, 2005). They define the accessible energy as the energy conventional turbines could generate in areas with a wind speed exceeding $7 \mathrm{~m} / \mathrm{s}$ at ten meters vertically, which is established as a lower limit in their feasibility study. Through analysis of readings from European and American meteorological stations, they discovered that only about $13 \%$ of the land area surveyed is suitable for production. Unlike a fission or combustion based fuel source, wind power is not globally viable due to these constraints. However, accessibility can be greatly increased if an array could be constructed to concentrate lower speed air streams into higher velocity jets.

To increase the accessibility of wind power and tap into this potential energy goldmine, engineers and inventors have worked to create such an array. The average land-area wind speed globally is about $3 \mathrm{~m} / \mathrm{s}$, well below the necessary minimum to allow power generation. A wind concentrator that could take an air mass at this speed and accelerate it efficiently to the required speed would open another $37 \%$ of global land area to the possibility of wind power.

Statistically, another half of the world's landmass must have wind speeds below this average. Louisville is one such area, with an average wind speed of less than $4.5 \mathrm{~m} / \mathrm{s}$ at ten meters (Archer and Jacobson, 2005). Previous attempts to design concentrators, as seen further in the literature review, often failed due to a lack of optimization because, before recently, no software could adequately represent or predict wind flow around a 
structure. The cost to optimize an untested design was prohibitive, as each prototype would need to be built from scratch. However, through the use of computational fluid dynamics (CFD) software, one may create many iterations of a design for minimal cost.

There is currently no significant wind power generation in Louisville due to the low average wind speeds. Wind concentrators can fill a niche for this region and other regions with similar wind speeds long left empty by prohibitive costs.

This thesis describes in detail the design of this wind concentrator and presents the CFD results that show its effectiveness for accelerating wind speed.

\section{Objectives:}

The objectives of this research were to:

1. Use CFD to model the wind concentration device.

2. Compare maximum velocities achieved for various designs of a center flow straightener, vortex breaker, and pressure relief slits in the device. 


\section{Literature Review}

\section{A. Computational Fluid Dynamics}

Computational fluid dynamics (CFD) is a field developed to apply material and energy balances in a calculation-intensive manner to determine the heat and mass transfer characteristics of a system. It is a fairly new science, as the computational power required to generate such data is only now becoming widely available. For these programs, scientists define a fluid as a substance with a microscopic structure that offers no opposition to shear forces. Rather, the shape of the substance will deform with the force and flow within its container. Fluid flow can be understood and predicted by observing the properties of the fluid at two or more boundary conditions, such as the ends of a pipe, and applying principles of conservation of momentum and mass to determine unknown variables at either end from the properties at another boundary (Ferzinger, 2002).

For a system such as an elbow in a pipe, one may determine the pressure and mass transit through the inlet and outlet planes of the pipe -for example, a radial crosssection of the pipe where measurements are taken- through the previously stated conservation principals. However, one knows almost nothing about the fluid flow between these two boundaries. To know more, one may take more cross sections, transforming the elbow into a sequence of conic sections, with calculations of mass and

momentum transfer occurring in each segment, or cell. One may use differential equations to view the overall profile of properties along the length of the elbow. 
However, this model would assume the fluid properties are uniform along the radius of the pipe, which is not accurate.

To correct this erroneous assumption, an engineer would typically do what he or she did before - add more boundary planes. In the conic sections the pipe walls are assumed to have zero transfer of mass or energy through them. They are made of an impermeable metal existing in a vacuum. One may then create a series of half-conic sections by bisecting the elbow with another plane. But what about the fluid within the cells? Fluid that is not contiguous with a wall boundary must have a fourth plane to form a new solid within which a calculation may take place - a tetrahedron. Therefore, after taking this to its logical conclusion, one would end with an arbitrary number of tetrahedral cells forming the approximate shape of the elbow pipe (Chung, 2002).

The computations become more elaborate and cumbersome as one adds more boundary conditions per cell. Additionally, the computing power required to define the fluid properties at each plane become far removed from the reach of the human mind. The only bits of information to work with are the data at the initial two planes of entry and exit and the knowledge that the model walls are impermeable to fluid flow and therefore mass or momentum transit. A computer programmed with this information may guess the mass and momentum transit values at the planes of the cells which contain one face bordering the boundary layers. It may then guess those of the adjacent cells who share at least one face with the boundary cells. This continues until all the cells have values. This is then repeated using the calculated guesses until convergence is achieved.

All this is to model a single pipe elbow in an isothermal system with no turbulent or external forces. Technology has provided ways to incorporate these forces which 
allows engineers to create "real world" models for fluid systems. For modeling forces through a wind concentrator, one would use a equation set that incorporates friction and turbulence into the momentum balances, as well as gravity (although because a pocket of air exists in an environment of equal density, this may be safely ignored). Additionally, it must include robust equations of state that incorporate factors such as density change with temperature and pressure in its calculations. The realizable k- $\epsilon$ model provides these features, and will be discussed further.

\section{B. Realizable k-c Model}

The modeling suite must go above and beyond what is necessary for simple conservation laws to model a turbulent system in a CFD, but must still factor these principles into their calculations. The k-€ equations for which the model is named will be discussed in detail, and an understanding of the basic continuity equations is necessary to their discussion. These allow analysis of a system such as a wind concentrator in which heat transfer and density change are negligible and flow is turbulent (Lamox et al., 1999).

The simplest of the conservation equations is the continuity equation, which is used to model the conservation of mass in the ANSYS CFD suite:

$$
S_{m}=\frac{\partial \rho}{\partial t}+\nabla *(\rho \vec{v})
$$

Equation 1 shows the form of the continuity equation detailed in the ANSYS theory manual (ANSYS, 2009). $S_{m}$ represents the rate that any net mass added to the system. In many cases for the system, input will be equal to output or eventually reach a steady state. This allows the CFD program to converge onto a constant and meaningful result. 
$\rho$ represents density, and $\vec{v}$ is the velocity vector. In a system with little to no density change, the derivative density term becomes negligible and may be divided out in a steady state system where $S_{m}$ is zero. Lastly, the $\nabla$ is the del operator:

$$
\sum_{1}^{i} e^{i} d i
$$

For a wind concentrator which operates in an open environment or a pipeline pumping a hydraulic fluid, steady state is maintained and there is no appreciable density change. Therefore, the continuity equation for the system becomes:

$$
0=\nabla * \vec{v}
$$

This equation is by the commonly used constant-density form of the continuity equation, shown as equation 4.6 in Unit Operations of Chemical Engineering (McCabe et al., 2000). This equation is not robust enough to accurately predict fluid behavior in most situations. External forces will act upon the system and nearly any system will have the force of gravity. To model this, another equation is incorporated into the CFD suite: the Navier Stokes equation (Chung, 2002):

$$
-\nabla p+\nabla * \bar{\tau}+\rho \vec{g}+\vec{F}=\frac{\partial}{\partial t}(\rho \vec{v})+\nabla *(\rho \vec{v} \vec{v})
$$


$p$ is pressure, $\vec{\tau}$ is the stress tensor, $\vec{g}$ is the acceleration due to gravity, and $\vec{F}$ is a net external force. The ANSYS suite uses equation 4 as its momentum balance equation. This equation simplifies further in a constant pressure, constant density, isothermal steady state system with negligible gravitatal forces (such as neutrally bouyant systems):

$$
\nabla \bar{\tau}=\nabla *(\rho \vec{v} \vec{v})
$$

While equation 4 accounts for compressibility, it does not take into account temperature changes and energy transfer - for this, ANSYS has prepared a third conservation equation:

$$
\frac{\partial \rho}{\partial t}(\rho E)+\nabla *(\vec{v}(\rho E+p))=\nabla *\left(k_{e f f} \nabla T\right)+S_{h}
$$

Where $k_{\text {eff }}$ is the thermal conductivity of the substance, $\mathrm{E}$ is an experimentally determined sensible enthalpy provided by ANSYS, T is temperature, and $S_{h}$ is the heat added to the system. $S_{h}$ is user-defined, and allows one to create boundary conditions that transfer heat realistically, such as a heat exchanger or hot plate. This equation simplifies further with the assumptions listed with equations 5 and 3 and no net energy flow:

$$
\nabla *(\vec{v}(\rho E+p))=0
$$


These equations fail to account for the effects of turbulence in systems where turbulent forces are dominant.

Fluids begin to act in non-fluidic ways at high Reynolds numbers, such as in a wind concentrator system. Shear frictional forces in the fluid curl the momentum vectors creating swirls called eddies. To account for the loss of forward momentum to eddies, the realizable k- $\epsilon$ model employs its namesakes $-\mathrm{k}$, which represents the turbulence kinetic energy, and $\epsilon$, which represents the rate at which this turbulence dissipates.

$$
\begin{gathered}
\frac{\partial}{\partial t}(\rho k)+\frac{\partial \rho}{\partial x_{j}}\left(\rho k u_{j}\right)=\frac{\partial}{\partial x_{j}}\left[\left(\mu+\frac{\mu_{t}}{\sigma_{k}}\right) \frac{\partial k}{\partial x_{j}}\right]+G_{k}+G_{b}-\rho \epsilon-Y_{M}+S_{k} \\
\frac{\partial}{\partial t}(\rho \epsilon)+\frac{\partial}{\partial x_{j}}\left(\rho \epsilon u_{j}\right)=\frac{\partial}{\partial x_{j}}\left[\left(\mu+\frac{\mu_{t}}{\sigma_{\varepsilon}}\right) \frac{\partial \epsilon}{\partial x_{j}}\right]+\rho C_{1} S \epsilon-\rho C_{2} \frac{\epsilon^{2}}{k+\sqrt{v \epsilon}}+C_{1 \epsilon} \frac{E}{k} C_{3 \varepsilon} G_{b}+S_{E}
\end{gathered}
$$

Where $G_{k}$ is the generation of kinetic energy due to the velocity gradient in the system, $G_{b}$ is the generation due to buoyancy (which is negligible in a constant density system), $E$ is the value from equation $6, Y_{m}$ is the turbulent energy generation due to compressibility experimentally calculated by ANSYS(again, negligible in a nearly hydraulic system), the $C_{1-3 e}$ terms are tuning numbers that vary depending on the system dynamics, and the $S$ terms are user defined inputs that allow different boundary conditions to be modeled (ANSYS, 2009). These equations simplify further in the system described for equations 3,5 and 7 :

$$
\frac{\partial}{\partial x_{i}}\left[\left(\mu+\frac{\mu_{t}}{\sigma_{k}}\right) \frac{\partial k}{\partial x_{i}}\right]+G_{k}+G_{b}=\rho \epsilon
$$




$$
\frac{\partial}{\partial x_{i}}\left[\left(\mu+\frac{\mu_{t}}{\sigma_{k}}\right) \frac{\partial \epsilon}{\partial x_{i}}\right]+C_{1} \frac{\epsilon}{k}\left(G_{k}+C_{3 \varepsilon} G_{b}\right)=C_{2 \varepsilon} \rho \frac{\epsilon^{2}}{k}
$$

ANSYS calculates the variables of the five equations at each cell boundary through each iteration of its calculations. The realizable k- $\epsilon$ model is relatively new, and improves upon the basic model by turning the $C$ constants further, producing more accurate results. The results are best for linear or circular jets in turbulent fluids with little compression. It is ideal for modeling wind concentration, and is relatively light with regards to calculation, making it best for optimization work, which often involves multiple trials of several designs.

\section{Wind Concentration and Low Velocity Turbines}

Wind turbines are the product of convergent technological evolution in most agrarian societies. The technology that later developed into the first windmill arose out the need to grind large amounts of grain. An efficient method of grain grinding is a necessity in any society with such a staple food source. As a society grows so too does its need for food and therefore the need for manual labor. While today, carbohydrate products such as bread are among the cheapest food products in terms of caloric density, in ancient times the preparation of grain was a limiting factor in food production.

Historians assert that it was the Egyptians who created the first windmills in 3,000 BC. Regardless of its first inventor, the technology sprung up in most agrarian societies within the next millennium. Farmers of this era devised two families of techniques to boost their ability to process grains. The first was a horizontal mill. Here, a beast of 
burden would pull a lever attached to a grinding wheel at the other end of the lever, marching in a circle around a central pestle, effectively grinding larger quantities of grain. The other design was a bit more complex. Here, water would propel a vertical wheel, which through the action of gears would perform the grinding. These two archetypes later evolved into the two types of turbines seen today - the vertically rotating turbine and the horizontally rotating turbine - replacing either animal or water power with wings to capture the power of air (Hau, 2006).

Wind-capture technology saw little improvement until the end of the $19^{\text {th }}$ century AD. There was no incentive - the mills performed their tasks. However, as more and more major cities gained power plants, rural areas were slow to advance due to the large land and resource requirements of such facilities. Around this time, farmers began to replace mill wheels with dynamos in fallow windmills (Hau, 2006). The beginnings of farming wind for electrical energy mirror the goal for it today: personal energy generation. However, in a modern setting, engineers wish to allow even low-wind zones to profit from such advances.

From the two families of windmills, two families of energy collection technologies have arisen. The first is the collection of vertical axis rotors. These consist of three primary rotor designs: the Savonius Rotor, the Darrieus Rotor and the H-Rotor (See Figure 1). The Savonius is S-shaped when viewed from above. This shape allows it to act like a stretched cup-anemometer. A single sheet of metal is bent to form the Sshape. Each curve of the $\mathrm{S}$ acts as a cup, catching a breeze and turning the axel. This design has no direction-of-interest, unlike horizontal axis rotors, and will catch wind coming from any direction. 
The Darrieus rotor is less bulky than its Savonius cousin. In this design, the blade of the rotor is shaped like a Mobius strip held vertically, creating a contoured loop. While it does not "cup" the wind like a Savonius, the curvature of the strip causes one side to present less resistance during a gust of wind, forcing the blade, and therefore the axel to spin. This design uses comparatively little resources, but captures less energy. Additionally, once this type of rotor stops spinning, it may be reluctant to independently begin moving again due to an unfavorable wind direction. It does have the lowmaintenance aspect of its family still - it will capture any horizontal wind source.

The last major phylum of vertical axis rotors is a derivation of the Darrieus. The H-rotor replaces the loop with two or more vertical airfoils. These are held onto the vertical axis by horizontal supports, creating the illusion of the letter $\mathrm{H}$ when viewed from the front. The lift generated in this design will rotate the axel, generating electricity.

The H-rotor is the cutting edge in the design of the vertical axis - an airfoil offers a flexible design with plenty of room for improvement. In fact, if you were to take these airfoils, taper them and attach them directly to the axel, and gear that axel to rotate horizontally, you would get a general design for the staple of wind power generation: the conventional windmill. The first wind powered generators were designed off of unused horizontal axis windmills as mentioned above. This grants a lineage of design practices and advances. Additionally, advances in aircraft propellers and flight technology in general have the synergistic effect of improving the action of windmills. A distinct downside to this type of power generator is the directional needs. This can be corrected with motors and sensors, but is ultimately more labor intensive. However, this disadvantage grants the single most important advantage of horizontal axis: there is no 
need for radial symmetry. A huge array of construction options becomes immediately available with this revelation, from stabilizing wings to elaborate wind concentration devices (Hau, 2006).
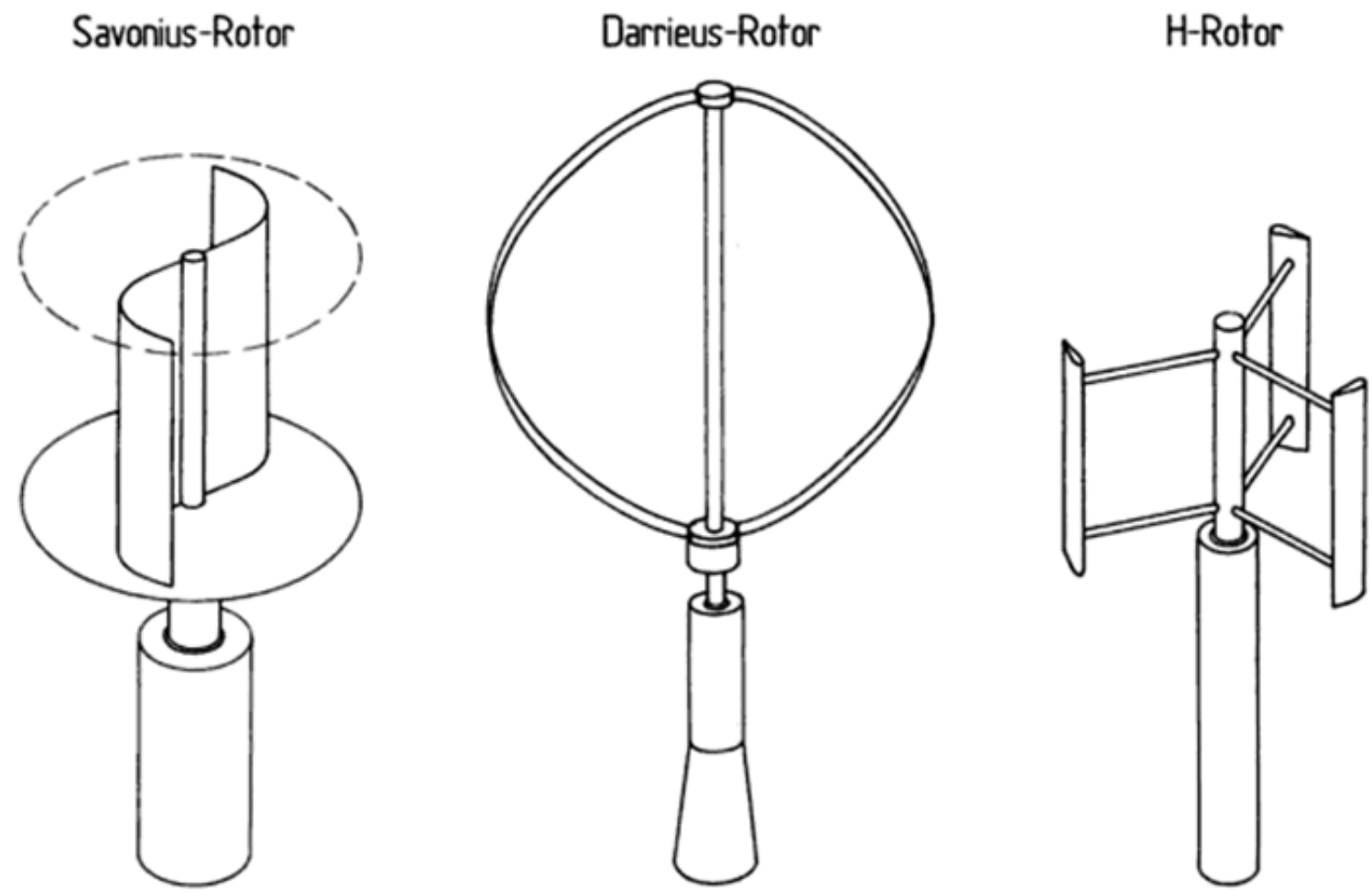

FIGURE 1. Various Vertical Axis Wind Turbine Designs (Hau, 2009)

A vertical axis rotor has little means of wind concentration. The necessity of radial symmetry keeps modification to a minimum. Forgoing one direction in favor of wind concentration removes this important benefit. A rotor has a certain threshold speed before it will become useful. With vertical axis generators, it is usually only nature who may engineer this. Wind concentration is a taboo area for vertical axis generators. However, they may be adapted for low velocity conditions by attempting to lower their threshold speed to allow their use.

An example of such a design is shown in the patent application literature for a novel device incorporating aspects of several types of vertical axis rotors. The Ultimate 
Wind Turbine System, shown in figure 2, patented by Edward Davis, is a device that incorporates elements of the Darrius and Savonius Rotors to form a turbine that will turn

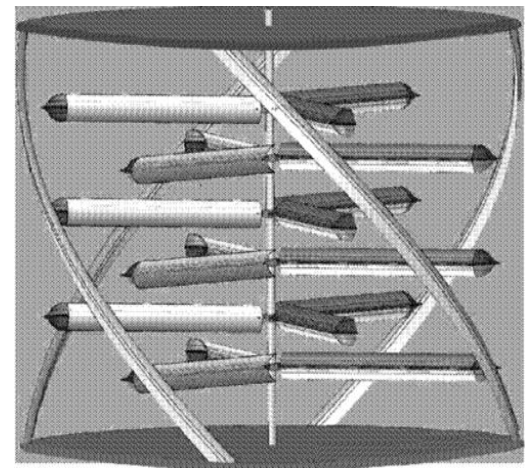

FIGURE 2. Ultimate Wind Turbine (Davis, 2010) at low velocities. It has four outer blades in the style of the Darrieus rotor between two disks that serve as rotating platforms, resembling a merry-go-round or an old-fashioned lawnmower blade.

There is also a pole through the center of the design, from which many troughs extend. There are shaped like spades on the wayward side, forming a design which will capture wind on one side and allow it to pass easily on the other. These flank the pole, forming an assembly with a high surface area and wind transparency (or low resistance when the wayward side is facing into the wind) (Davis, 2010).

This design captures more energy than a Darrieus rotor, without the heavy resistance on the wayward side that prevents Savonius rotors from functioning at low velocities and presents difficulties when beginning to spin. The most important proposed innovation is a novel set of magnetic bearings in the device which generate incredibly low amounts of friction.

With a base requiring about a square meter of land investment, his design is meant to be applied in tight urban environments on high-rise roofs. Bill Becker, an engineer, has developed another similar design integrating the Savonius and Darrieus designs (Becker, 2010). In his, a single unbroken Savonius blade twists around a center 
axel, with Darrieus rotors spiraling as in Edward's design. The "Aeroturbines" are commercially available, and are helping urban buildings approach energy independence. The common theme among these two vertical axis rotors is a utilization of the maximum available surface area within the Darrieus rotor. The Darrieus rotor forms a boundary for potential volume utilization, generating a motive force that may rival a Savonius design. When a Savonius rotor is added within it, an engineer must balance the benefits of the extra wind capture with the reduced throughput inside the Darrieus construct. Finding the optimal size for the Savonius rotor within each design produces the best design, as we see in the two schematics represented (Becker, 2010).

Some radially symmetric structures may act as wind concentrators for vertical axis rotors. Occasionally, when other solar energy capture operations are unavailable, a large metal sheet will act as an energy capture mechanism for a wind generation project. This sheet would have a tube rising from the center and be elevated a few inches above the ground. As solar radiation heats the sheet, the air beneath it heats, becoming less dense and moving through the tube in the center of the assembly. This area of rapid air flow can be fitted with a vertical generator or a more conventional turbine. Still, designs of this nature are better classified as solar power than concentrated wind power.

For a vertical axis rotor, one of the best understood means of concentration is to nest one rotor within another. This is shown above in Figure 1, as two of the low velocity designs use a Darrieus rotor to act as a vortex generator of Savonius rotors inside. When even this is not enough, engineers may design a super-structure around the inner dynamo. Victor Walters created one such schematic, patented as "Walter's Whirl-Wind." 
It consists of two similar concentric assemblies of airfoils. The first is the outer right, with a diameter nearly triple the inside ring. This ring's purpose is to capture and propel air into the second ring, which used a dynamo to capture the momentum of the masses of air flowing into it. Despite being over fifteen years old, his patent and idea has done little within the industry. Concentric designs may provide a starting point for optimization, but do little for lowering the threshold of usefulness for a vertical axis rotor (Edwards, 1996).

For horizontal axis rotors, there is far more that may be done to improve the effectiveness of a land investment to wind energy. Engineers may use larger structures like ducts and cones without the symmetrical concerns related to vertical rotors. These structures either increase the effective area covered by the rotors or increase the incoming wind velocities.

Wind concentrators take four major forms. The first is a shroud for the rotor, much like the sheath over the tail rotors of helicopters, preventing incoming air from being batted away by the spinning rotors and allowing more of the momentum to be transferred. The second is a funnel at the outgoing end of the turbine, often used in conjunction with a shroud. This design, called a diffuser, appears counter-productive, as the entire device looks backwards (See Figure 3). However, through research, it has been shown that a solid funnel in front of a rotor will actually hinder air from through the device (which is why a working forward funnel design is so unique). Instead, a diffuser behind the rotor pulls air through the center of the device as it a current of air must either move through the rotor, or expend even more energy to accelerate and compress down the length of the device before rejoining the bulk air body beyond the funnel. 


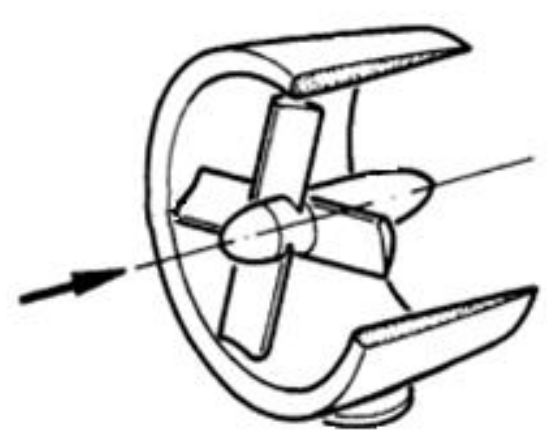

Shrouded wind turbine

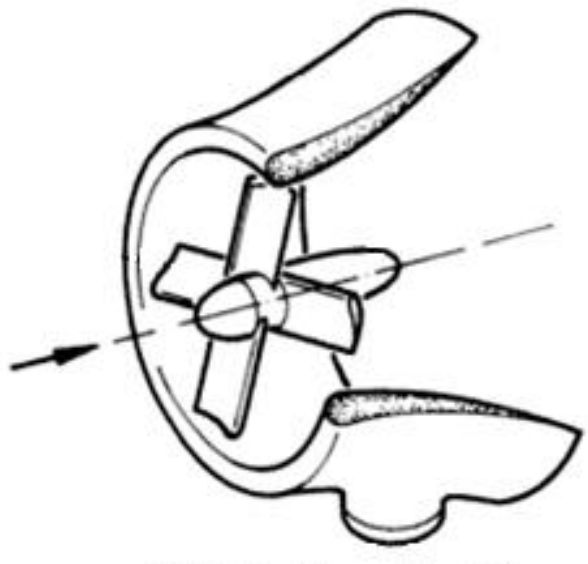

Wind turbine with diffusor

FIGURE 3. Turbine with Shroud or Diffuser (Hau, 2006)

In addition to a diffuser, an airfoil may be used behind the rotors to created increased airflow due to the principles of lift. The devices, called delta wings for their triangular profiles, extend behind a rotor. These devices are often coupled, forming a bilaterally symmetrical two-rotor generator. Their usage is limited however, as their predicted benefits do not exceed their prohibitive costs and large space requirements. The last family of designs contains futuristic looking rotors that use one stationary array in front of the actual power rotor. The stator here is much larger than the rotor, and contains a structure to "herd" more air molecules into the rotor. The prohibitive cost and mechanical difficulties associated with these has been a limiting factor in their deployment. However, for smaller generators, many mechanical problems become less important due to the reduced mass - these high tech turbines are becoming a focus for energy independence advocates (Hau, 2006). 
An example of a simple shroud would be a roof-top wind generator, much like the type Glen Kane investigated in a recent patent. A shrouded turbine is aesthetically pleasing, blending into whatever it is embedded into, and requires a small land investment compared to other designs. In Glen's schematic, the turbine would be part of an assembly that could be built as a unit and installed on the peaks of roofs, mimicking the appearance of a dryer exhaust or air-conditioning intake. The small size of the invention limits its usefulness, and the shroud does little work concentrating wind sources, further bounding this device to only be used in high-wind areas. Shrouds are rarely seen alone on generators - for the price of a shroud, a diffuser could be installed, slightly increasing the required land investment and decreasing the aesthetics, but nearly doubling the wind velocity coming through the rotor aperture (Kane, 2010).

This is shown by the commercial success of the WindTamer turbine as a personal power source. This WindTamer is a shrouded rotor with a diffuser at the wayward end. While it is aesthetically designed for marketability, it contains the important elements of a diffuser. The product appeals to those in moderate wind areas. Even in favorable locations, most turbines require a high relative elevation to run efficiently, often over a hundred feet. Through the use of a diffuser, the WindTamer will generate energy at just twenty feet, complying with most city-zoning laws. Diffusers have shown the most commercial success for small rotor designs, and have formed a lucrative sub-market within the wind power industry. The design is built from plastics with a high strength and low cost. Additionally, due to the relatively slow wind speeds the WindTamer will experience, the device can afford to be more "frilly" and complex with the concern of excessive sheer stress (WindTamer "Comparing Wind Technologies," 2010). 
Another example of this type of design is a turbine proposed by Clement Hiel and George Korzeniowski in 2005 with a similar design. This design has shown no success in the private sector however. The difference is not simply poor marketing. The reason WindTamer had an easy time securing their patent was due to the poor design of this turbine. Rather than use the diffuser behind the device, the inventors mistakenly put the diffuser on the front, forming an ill-fated funnel turbine, as described earlier. Because the inlet directions differed, each design constituted a novel idea. However, design changes and a well-designed intake cone may improve the prognosis for such designs (Heil et al., 2007).

The next family of designs involves those using delta wings to generator more powerful air currents. Delta wings use principles of lift to force air currents through a rotor, using a wing with a high angle of attack to promote a high velocity. However, according to Wind Turbines Fundamentals $2^{\text {nd }}$ Edition by Erich Hau, this family had such disappointing prototyped results that little investigation has been put into them. While theoretically sound, even the best designs would generate so much turbulence due to the attack angles on the wing that they would require. But this has not stopped inventors like John Loth from trying.

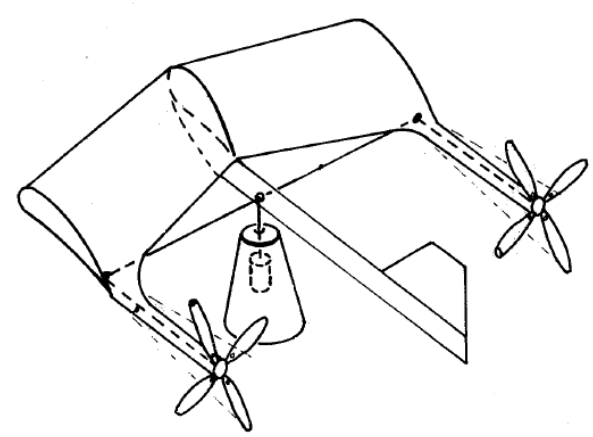

FIGURE 4. Loth's Turbine (Loth, 1977)
He submitted a patent in the mid-70s for a delta wing design (See Figure 4). It featured two rotors training behind a single solid wing, resembling a horizontally-laid staple, with a rotor at each end and an airfoil forming the 
center bar. This patent has even had time to become public domain, and still nothing has come of it (Loth, 1977).

The last family of rotors is also the most varied and has no distinct representative as with many of the categories above. The front-mounted diffuser mentioned above would actually fit more easily into this category, though it would be similarly ill-fated (Hau 2006). 


\section{Procedures}

\section{A. Geometry and Mesh Generation}

ICEM CFD version 12.1 is a robust modeling program with significant synergy with the fluent modeling suite. ICEM is useful for creating the geometry of the wind concentrator system, as well as to create the complete mesh. Additionally, ICEM may be used to generate meshes from solid geometries modeled in another program and imported. When meshing, ICEM divides these solids into a finite number of discrete elements, often consisting of tetrahedrons for 3D geometries; it can also generate hexahedral cells, or hybrids of either. ICEM can also generate a 2D surface mesh, consisting of either three or four-sided polygons. These meshes are then exported to Fluent version 12.1 for processing and analysis.

To model the two-dimensional flow of air through the wind concentrator, the concentrator geometry was assumed to be suspended in the center of a wind tunnel, with flow parallel to the ground. The diameter of the flow field is such that no boundary effects from its walls will affect the concentrator's efficiency. The concentrator array is located in the center of this cylinder shaped wind tunnel. The easiest way to construct the geometry for this radially symmetrical fluid space is to generate a "slice" of the geometry and sweep it around an axis to create a cylinder.

To create the slice, open the geometry tab from the toolbar and select "create point." In the menu this opens, select "explicit coordinates" as shown in Figure 5. This allows a point to be created from Cartesian coordinates. In this three axis system, the 
two-dimensional slice will be created on the X-Y plane and rotated through the third dimension. As such, all Z-values will be 0 for this part of the procedure.

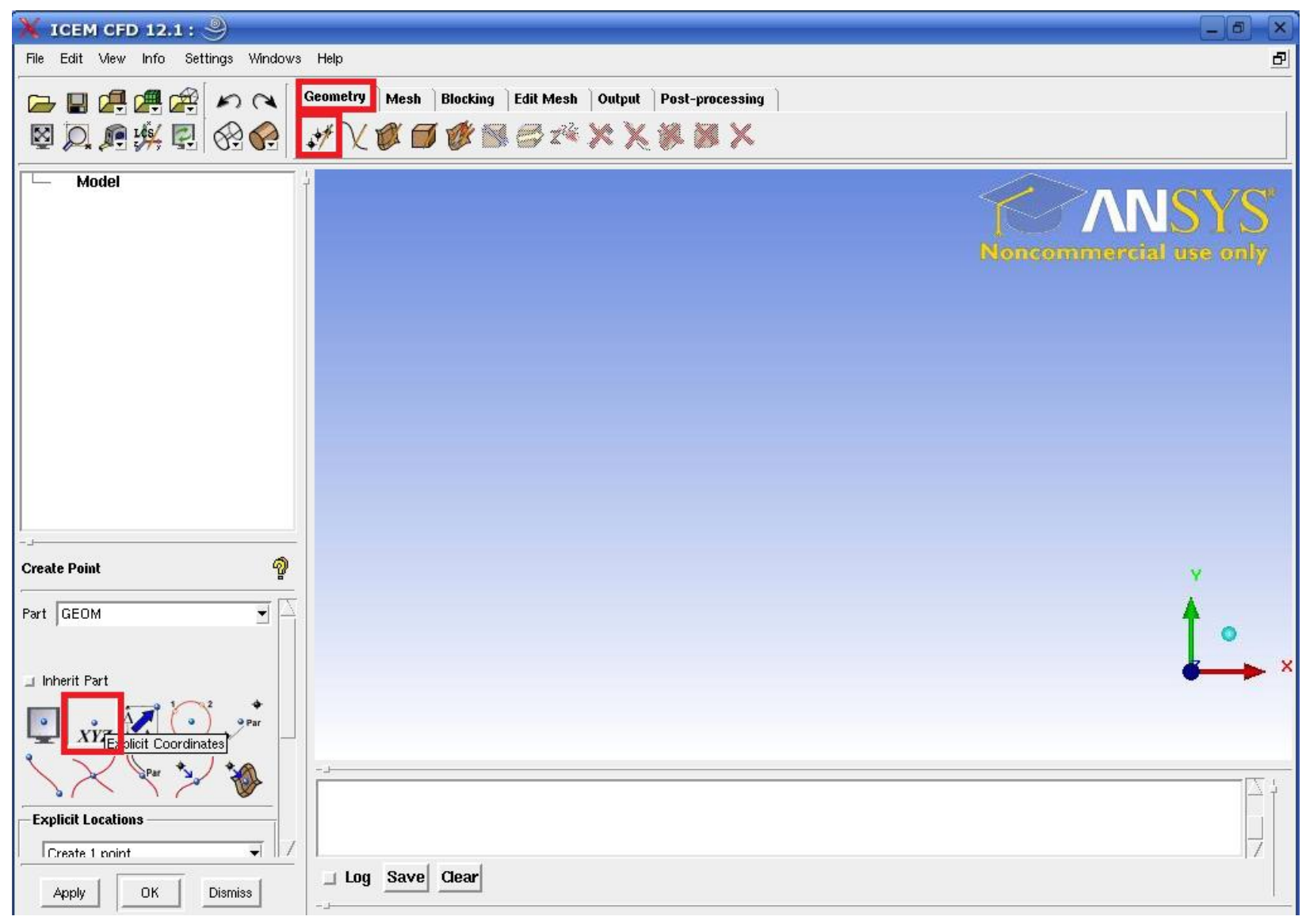

FIGURE 5. Explicit Coordinates

Now, to construct the radial portion of the wind tunnel, create the points $(-4.5$, $0.5,0),(-4.5,-4.5,0),(5.5,-4.5,0)$ and $(5.5,-.5,0)$ by entering the coordinates in the spaces provided in the "explicit coordinates menu" and press apply after each set has been entered. This will create a square of points. These unusual points allow the inside rim of the concentrator with a $0.5 \mathrm{~m}$ radius to be located at the origin.

To add lines to "close" the square, click on the "create/modify curve" button located next to the "create point" selection. Select the first option in the new menu, 
"From points." Connect the point, $(-4.5,0.5,0)$, to $(-4.5,-4.5,0)$ by selecting each in order, then confirm the selection with apply (a middle mouse click will also confirm). Next, connect $(-4.5,-4.5,0)$ to $(5.5,-4.5,0)$ and confirm; repeat with $(5.5,-4.5,0)$ and $(5.5,0.5,0)$

Now, using the point input methods from above, create the geometry of the concentrator and the internal cone. In models created for this thesis, this was done by plotting a set of 10-30 points for the interior profile of the concentrator, along with 10 for the cone. The line generation tool "from points" is robust in this program and will provide a smooth contour by selecting multiple points in succession before confirming the line. Multiple lines can be used to create sharp angles, such as at the rim of the concentrator to reduce turbulence. The central cones were modeled so that when mirrored with itself $180^{\circ}$, it would form a closed shape.

Now select "Create/modify surface," located next to "Create/modify curve," and select the "surface of revolution" tool. Set the start angle to 0 and the end angle to 180. At this stage in design, the model should resemble that in Figure 6. 


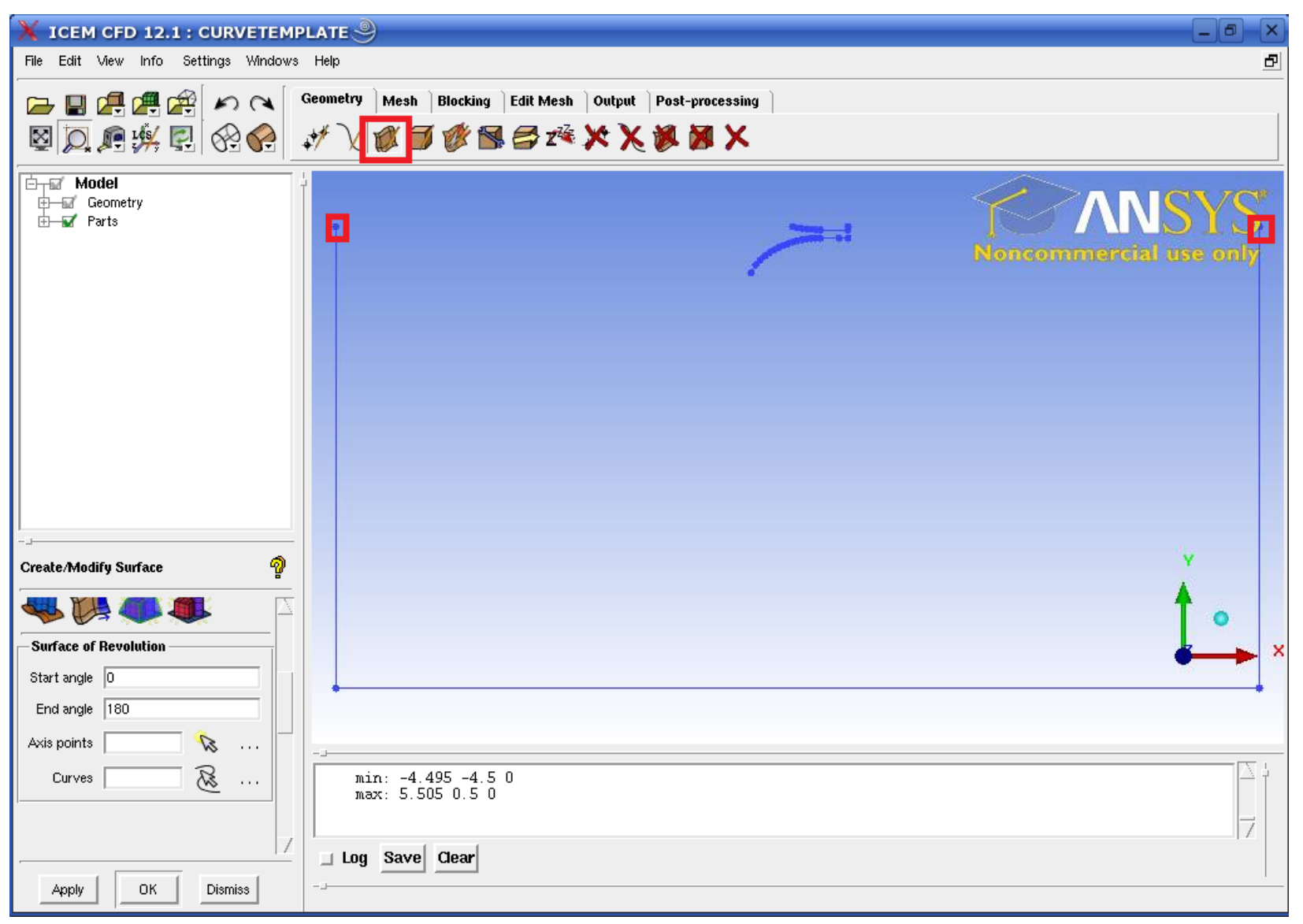

FIGURE 6. Surface of Revolution

When selecting axis points, select the two points boxed in Figure 6. These points are collinear with the center of the concentrator (assuming a proper $1 \mathrm{~m}$ internal diameter concentrator). When selecting curves, use the cursor to click and drag to highlight them all. Completing this operation will yield a half-cylinder. Repeat this operation on the same curves and axis, except with a start and end of 0 and -180 respectively. This will yield a full cylinder with curves at both $0^{\circ}$ and $180^{\circ}$. This allows better control when meshing the structure.

Now, right-click on the "Parts" menu of the "Model" drop-down menu. Select "create new" as in Figure 7. In the menu that appears, name the part "VEL_IN" (or 
similar) to label it as the mass transit inlet. Select "Entities" and left-click the mass inlet surfaces (the round end of the cylinder on the windward side of the concentrator). Select apply to create the part. Repeat this process on the leeward end, naming it "VEL_OUT" (or similar). The names are arbitrary, as boundary conditions will be defined for them later in Fluent.

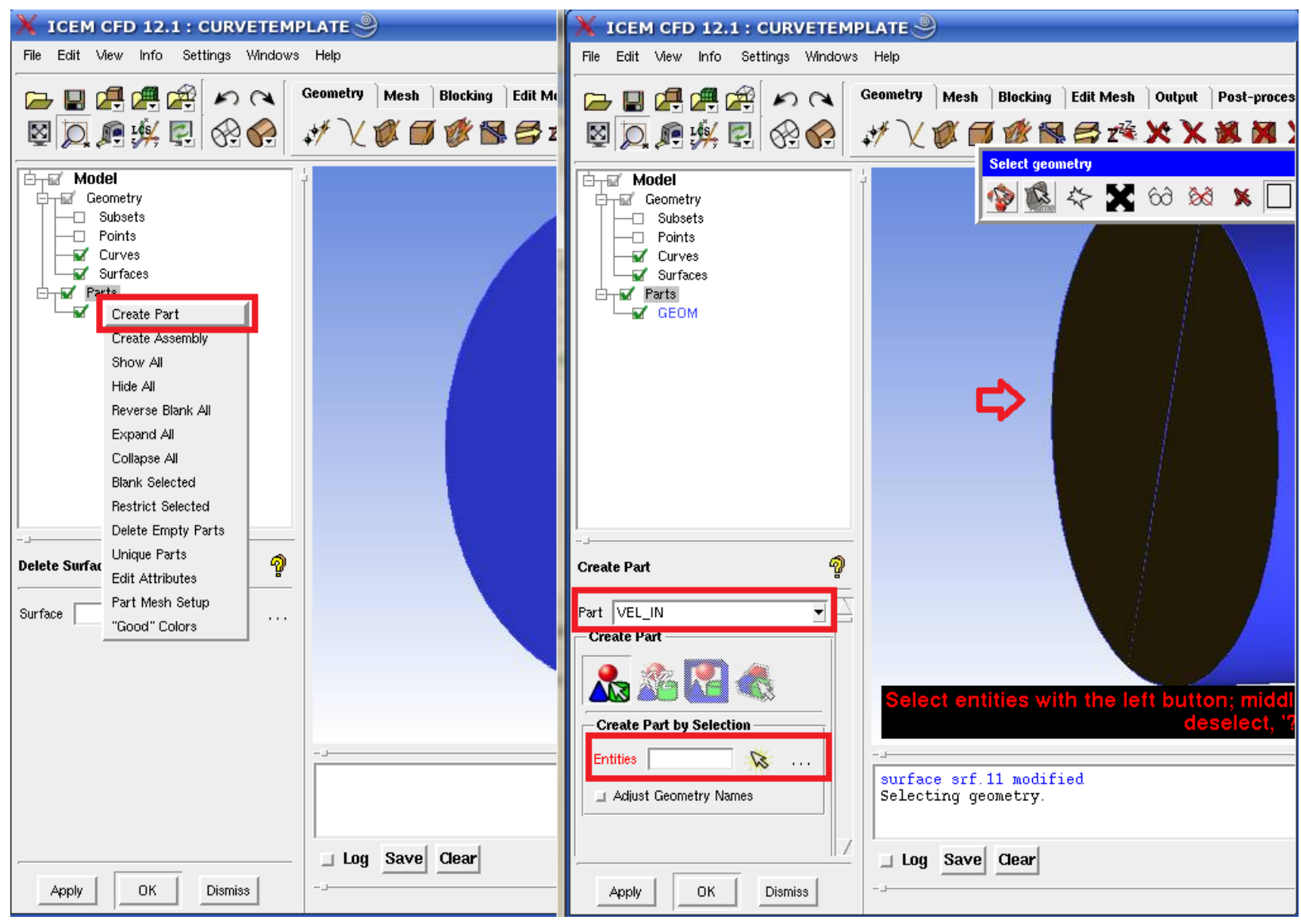

FIGURE 7. Part Creation 


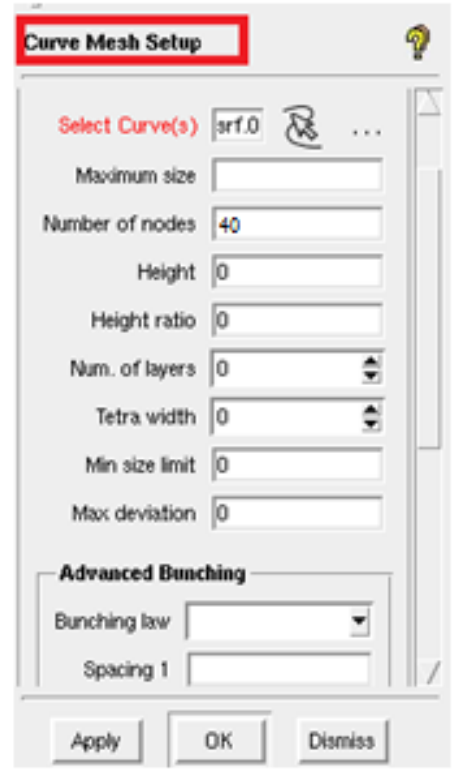

FIGURE 8. Mesh Nodes
After these two parts have been created (for a total of three), the model is ready to have a mesh applied to it. Select the "Mesh" menu, located next to the geometry tab. Select "Curve Mesh Setup.” For a well-sculpted mesh for these models, select all curves and set the number of nodes to 40 (see Figure 8). In models with large, skinny cones, one may have to individually select the cone curves and set the number to 100 ,

using the "advanced bunching" drop-down menu (selecting uniform) to ensure a good mesh size. For a model with a large cone, such as this example, a 40 node curve will suffice.

After the number of nodes per curve is established, the mesh may be generated. To generate the mesh, select the "Compute Mesh" icon, located at the right end of the "Mesh" tab. Select "compute" (located in the lower left corner) in the new menu without changing any other settings. If a dialog states that a mesh already exists, choose to replace that mesh with the new one by selecting "replace."

The mesh is now ready to be exported to fluent. To do this, first save the project. Next, select the "Output" tab from the top menu. Select the red tool box icon, whose tooltip reads "Select Solver." In the dropdown menu that results, select "Fluent V6." Now select the "Write Output" icon. Save the project if prompted, and open the 
corresponding .uns file for output. Name the file appropriately (in Figure 9, "EXAMPLE") and select "done." The mesh is now complete and ready to be loaded into Fluent.

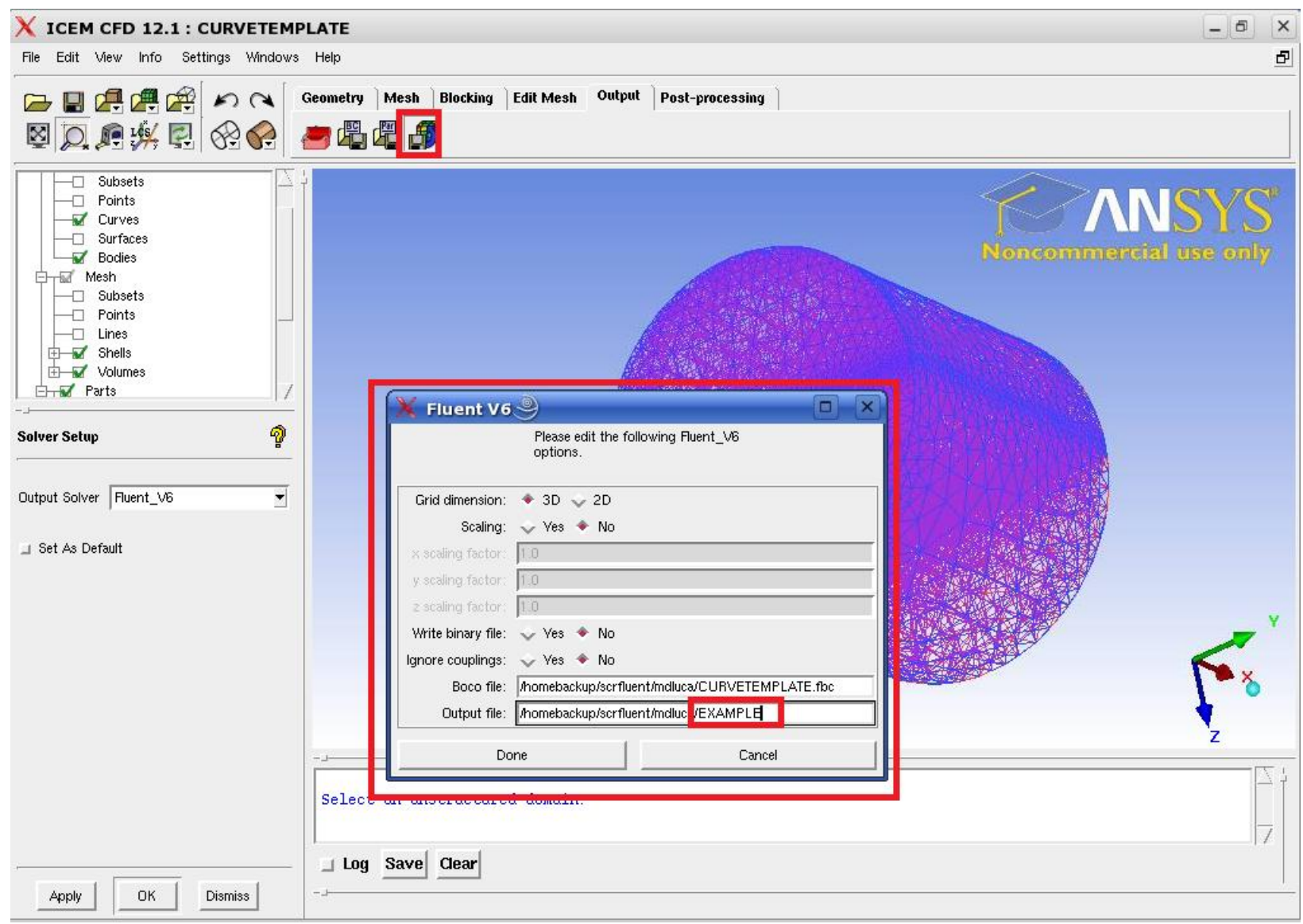

FIGURE 9. Output File

The output file is found at the location written in the "Output file" prompt (shown in Figure 9). 


\section{B. Problem Solving in Fluent}

To format the file in Fluent, begin by opening Fluent in 3D mode. Select "File" from the top toolbar, and select "Read"=>"Mesh..." Open the output file. The model will be properly scaled already, as Fluent's default unit of length is the meter. Now, become familiar with the Problem Setup menu on the left side of the screen. This is used to set the boundary conditions of the problem. The first thing to be modified is the "Models" selection. In this selection, the "Viscous" model should be changed from the default (laminar) to the Realizable k-epsilon ( 2 eqn) model. This is done by selecting "Edit..." with the "Viscous" text highlighted, and selecting "k-epsilon (2 eqn)" and "realizable" as shown in Figure 10.

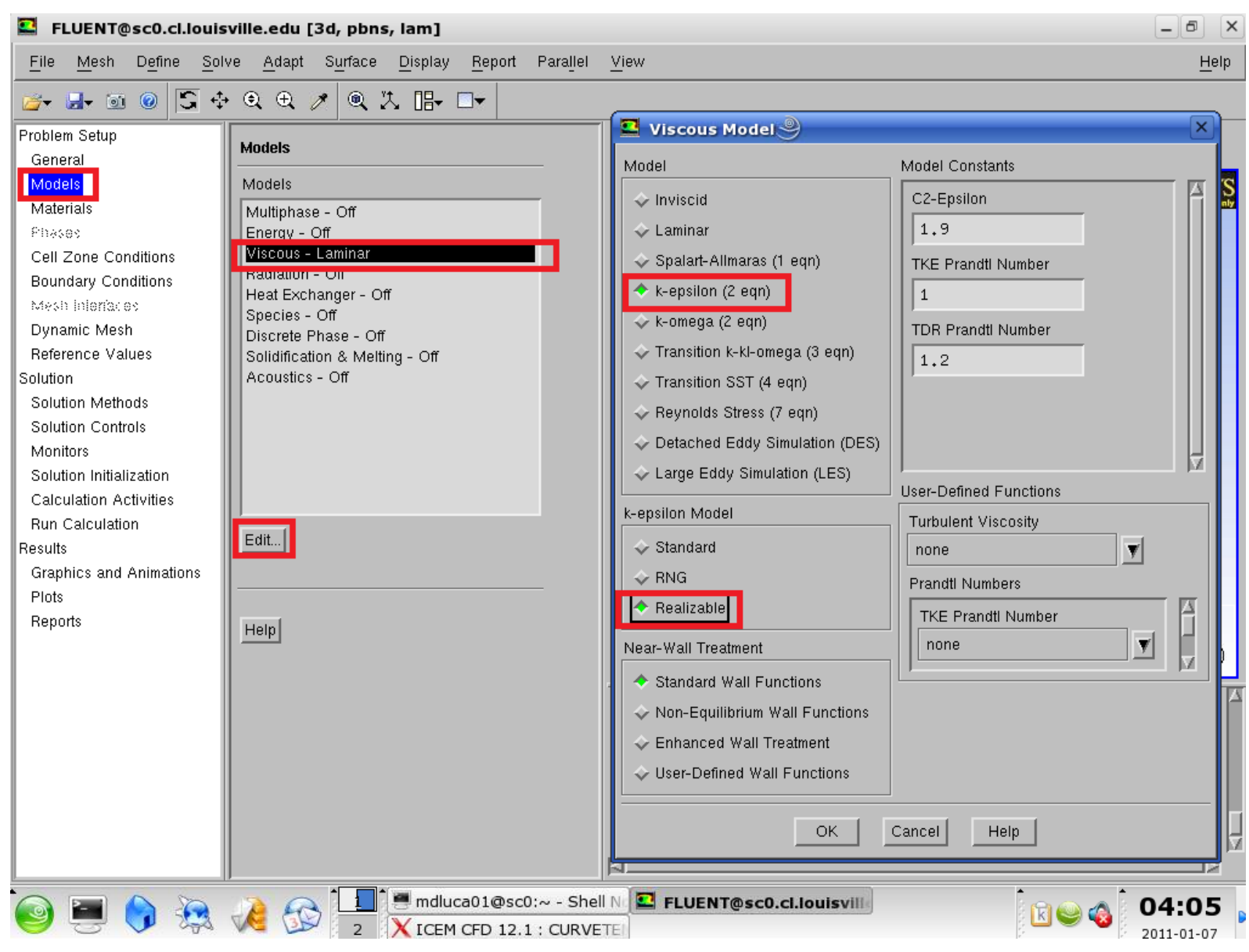

FIGURE 10. Viscous Model 
Select "Cell Zone Conditions" after this has been completed and ensure that Air is the fluid in the fluid-space of the model. This is done by selecting "Edit..." ensuring "air" is listed as the fluid in the drop-down menu titled "Material Name" (See Figure 11).

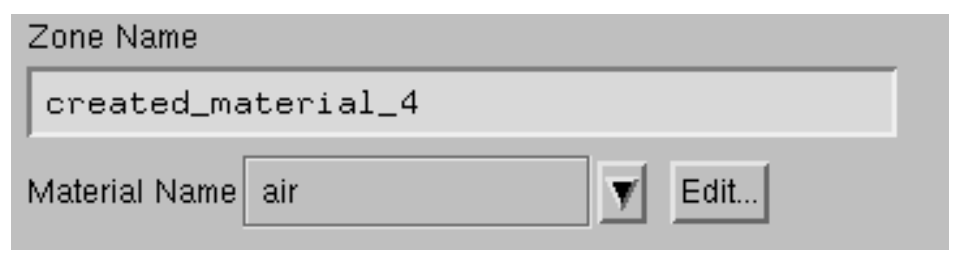

FIGURE 11. Material Name

Next, choose "Boundary Conditions." Fluent will display the parts of the model that were established in ICEMCFD: the internal mesh, the wall mesh describing the cylinder wall and the concentrator, the VEL_IN, and the VEL_OUT regions. The wall mesh will already be defined as a "Wall" boundary, and the internal mesh will be defined as "Interior." The remaining two zones should be defined as such: VEL_IN (or similar name depending on the nomenclature used when creating the mesh) should be a "mass flow inlet." This choice can be selected by highlighting VEL_IN and choosing this from the "Type" dropdown menu. This will bring up another menu, in which one may modify the mass flow rate. The mass flow rate at experimental conditions is $223.15 \mathrm{~kg} / \mathrm{s}$ as shown in Figure 12. 


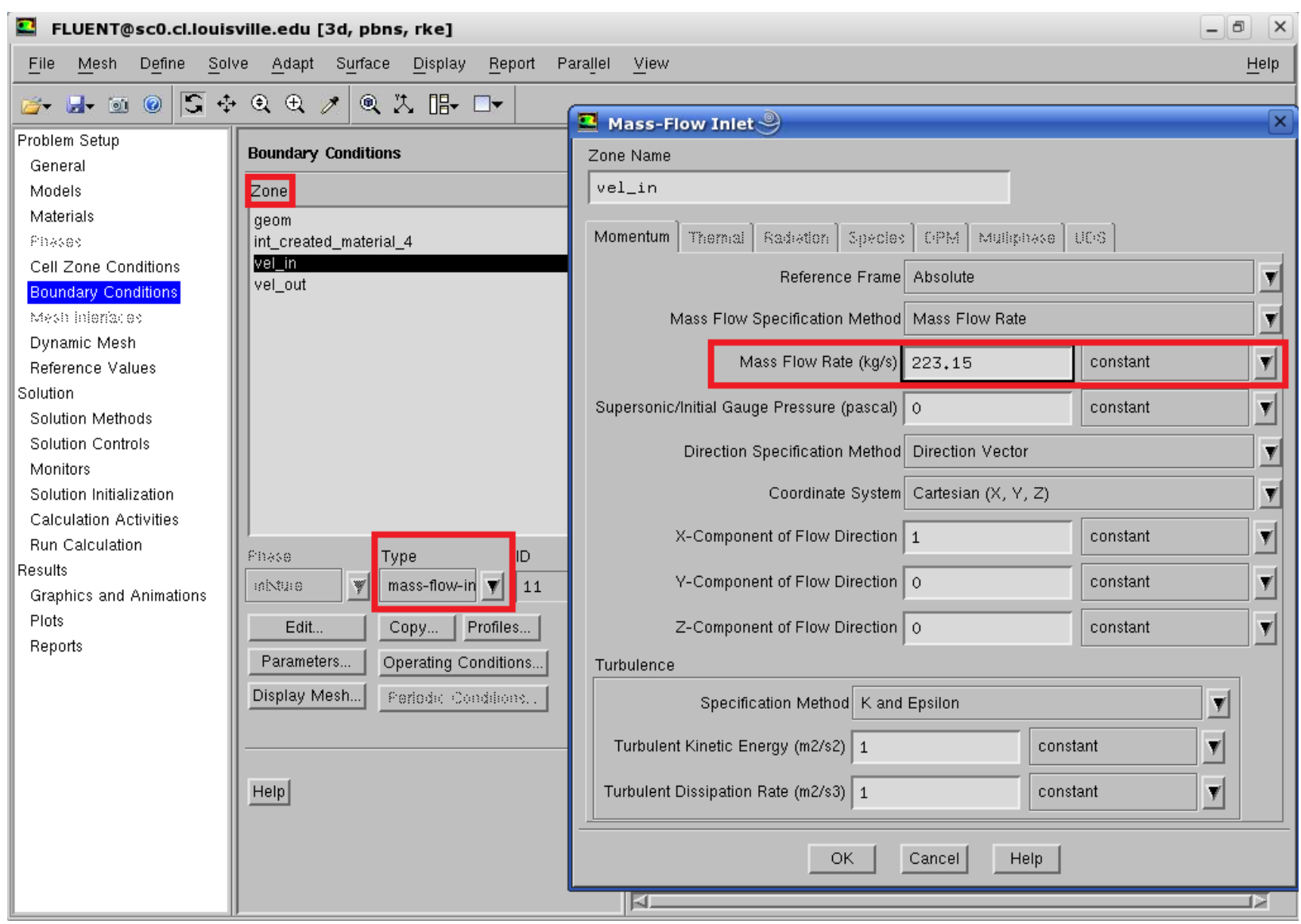

FIGURE 12. Boundary Conditions

Now highlight VEL_OUT, and select the type "pressure-outlet. This will bring up another window. Nothing should be changed here; simply select OK to close the window. This concludes the problem setup for this model.

Now begin to define the solution methods, listed in the "Solution Methods" menu. Here, select "coupled" from the "Scheme" dropdown menu, and select the options shown in Figure 13 in the remaining menus in the "Spatial Discretization" box. 


Spatial Discretization
\begin{tabular}{|l|l|l|}
\hline Gradient & Spatial Discretization \\
\hline Least Squares Cell Based & Second Order \\
\hline Pressure & Density \\
\hline Second Order & Third-Order MUSCL \\
\hline Density & Momentum \\
\hline Third-Order MUSCL & Third-Order MUSCL \\
\hline Momentum & Turbulent Kinetic Energy \\
\hline Third-Order MUSCL & Third-Order MUSCL \\
\hline Turbulent Kinetic Energy & Turbulent Dissipation Rate \\
\hline Third-Order MUSCL & Third-Order MUSCL \\
\hline Turbulent Dissipation Rate & Energy \\
\hline Third-Order MUSCL & Third-Order MUSCL \\
\hline
\end{tabular}

FIGURE 13. Spatial Discretization

Now, select "Solution Controls." Here, the explicit relaxation factors may be adjusted. These factors are used to determine how quickly the calculations may converge; too high and the calculation will often diverge, while if too low, a solution will never be reached. A good starting point for these values is 0.45 as shown in Figure 14 . These may be altered as needed. The other factors should not be changed. 
Select the "Monitors" option next and highlight "Residuals - Print, Plot." Press the "Edit..." button and increase the "Absolute Criteria" for all values to 1e-05 as in Figure 1. Press "OK" to confirm the alterations. This will allow calculations to continue until there is no variance in iterations greater than 0.00001 units.

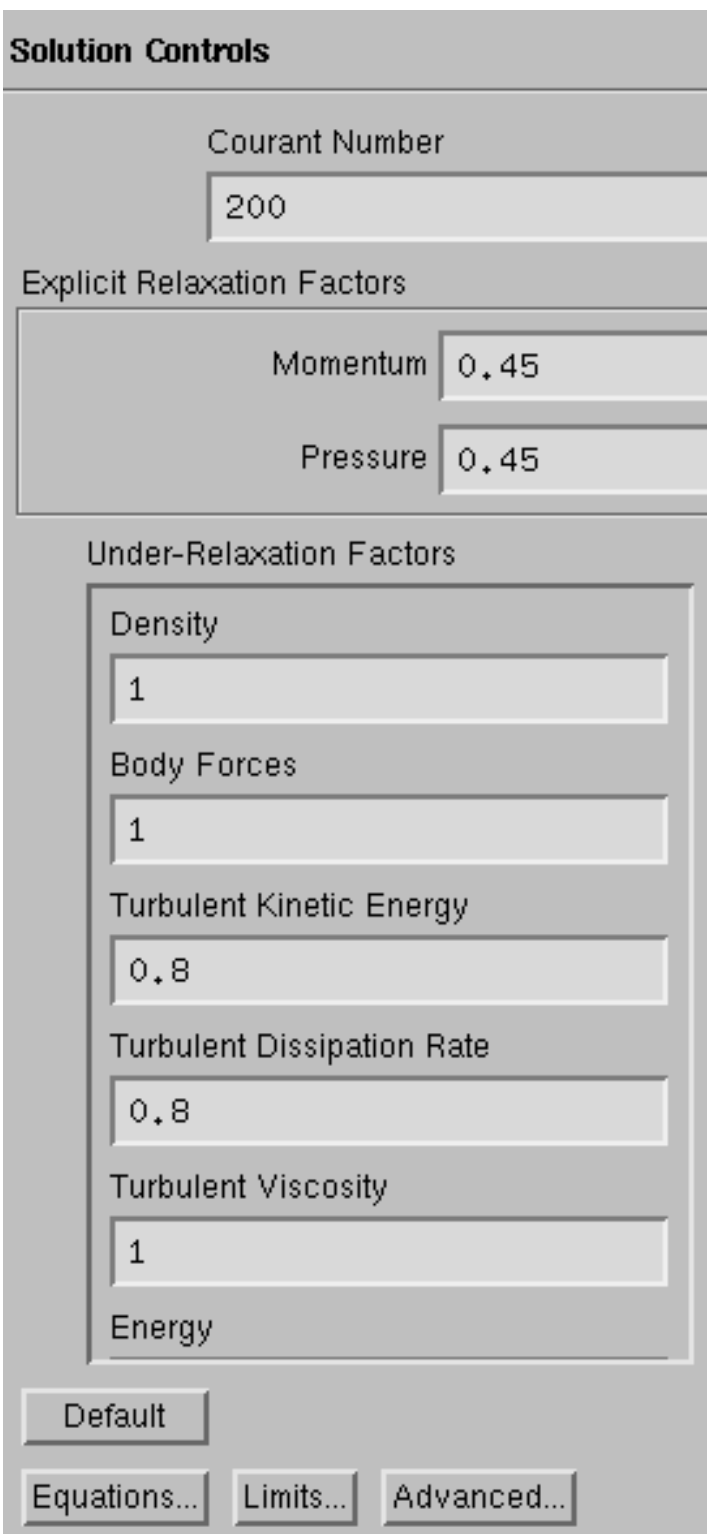

FIGURE 14. Solution Controls 
Select the "Solution Initialization" below "Monitors" now, and press the "Initialize" button to prepare the model for use (See Figure 16). Lastly, select "Run Calculation," input the number of iterations desired (generally 500 or more) and press calculate. After beginning calculations, wait until the calculation is completed by Fluent (signified by a window stating that convergence has occurred).

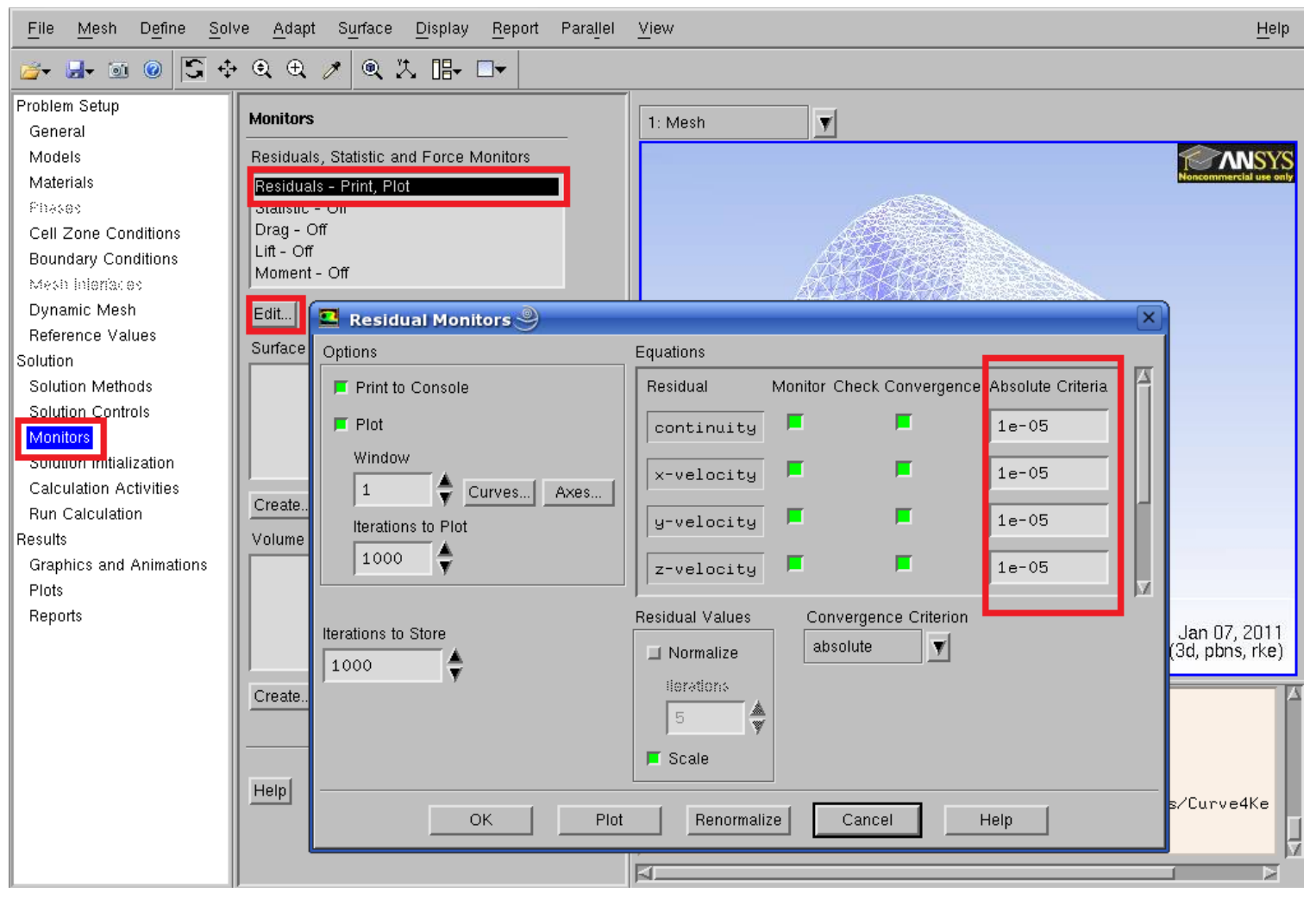

FIGURE 15. Monitors 


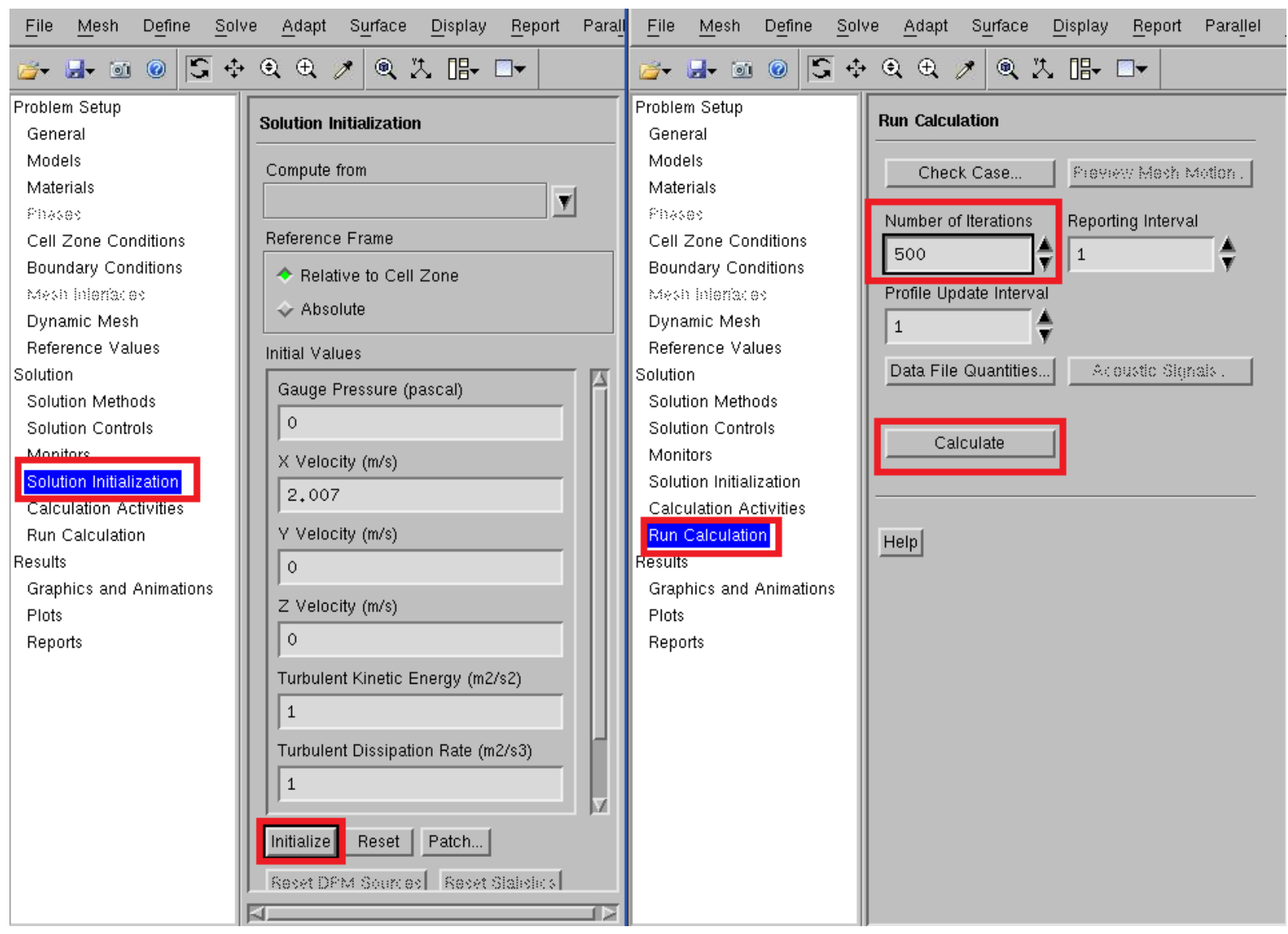

\section{FIGURE 16. Initialization and Calculation}

\section{Viewing Results}

To view the velocity and turbulence profile of the model, select Graphics and Animations" under "Results," highlight "Contours" and select "Set Up..." To best view the profiles, the plane used to transect the model should be along the $Z=0$ plane. To create this, select "New Surface" and choose "Plane..." from the dropdown menu (See Figure 17). Format the plane as shown in Figure 18, and press "Create" to create it. Select this plane from the "Surfaces" box, and select the desired property from the 
"Contours of" dropdown menu. While designing the concentrator, this procedure for model creation and data collection proved effective in all cases.

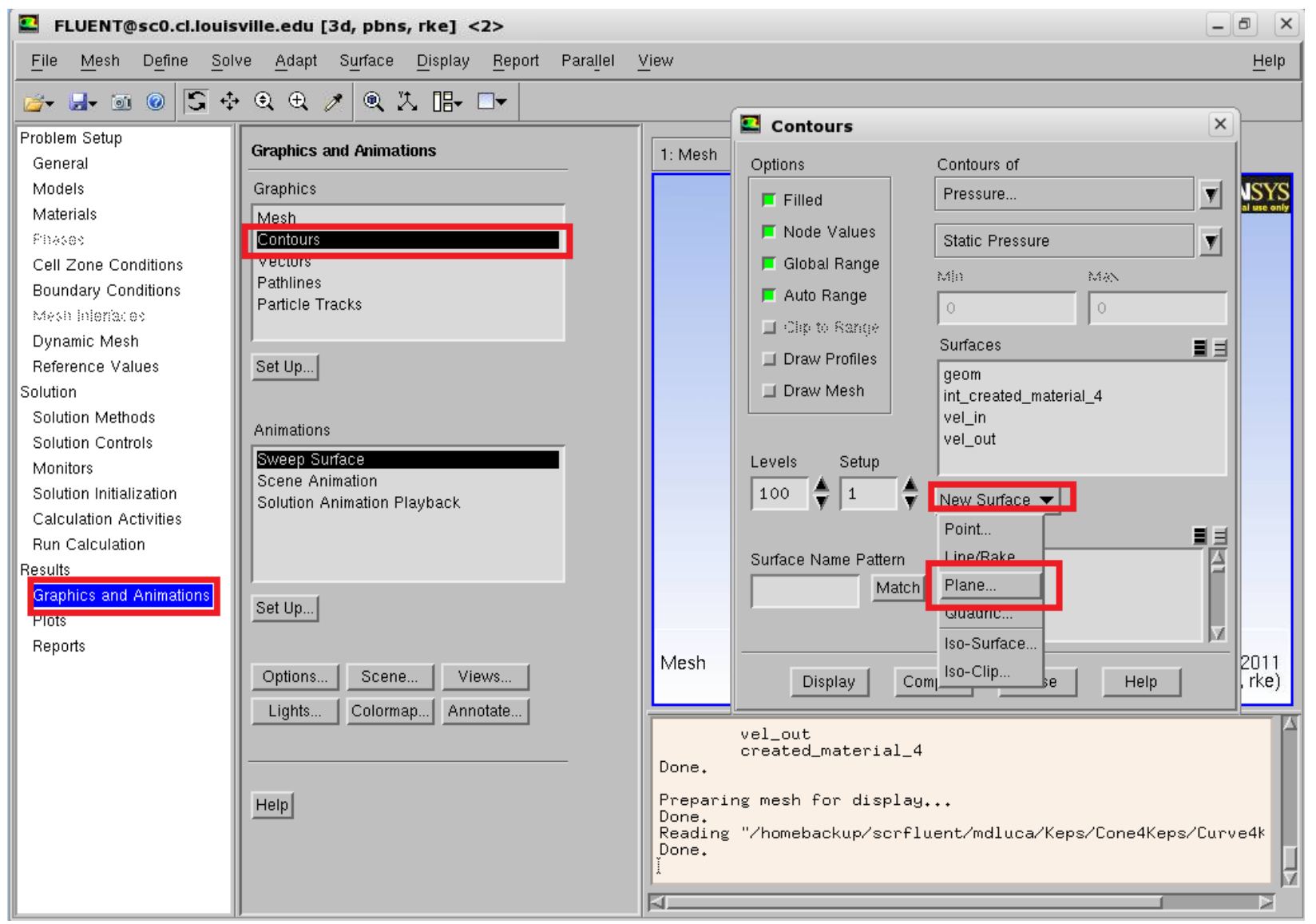

FIGURE 17. Graphics and Animations: Creating a plane 


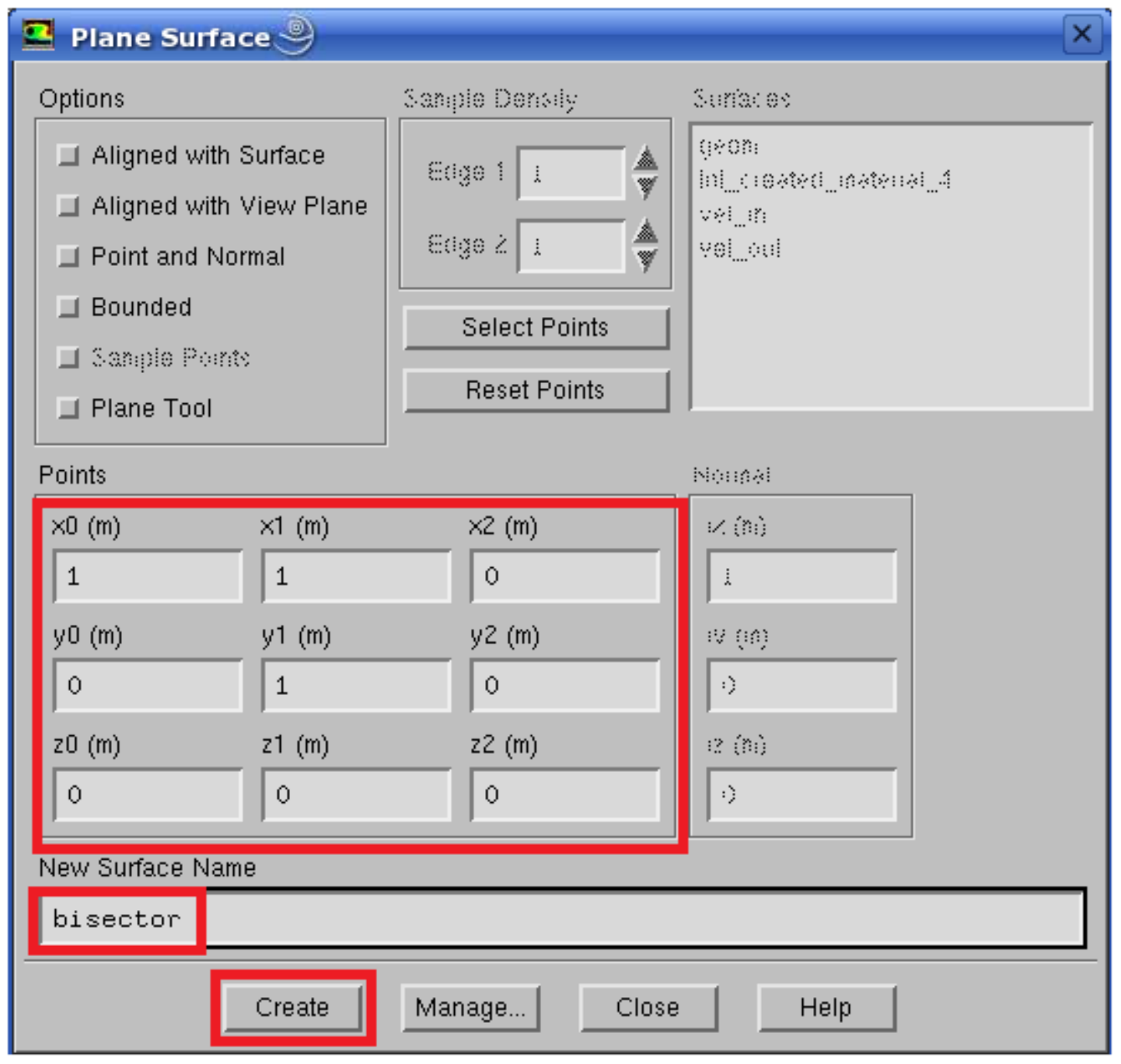

FIGURE 18. Graphics and Animations: Defining a plane 


\section{Results and Discussion}

The design evolved from a wholly holistic design, gaining new features and becoming better crafted with successive iterations. WEST Wind Power Inc. provided design features. The original design (Figure 19) provided a starting point for further improvement, which included testing of both a parabolic and a hyperbolic intake with a turbine housing and exhaust port. The key feature of the device was the intake; the turbine housing is reminiscent of the hooded designs shown in figure 3.

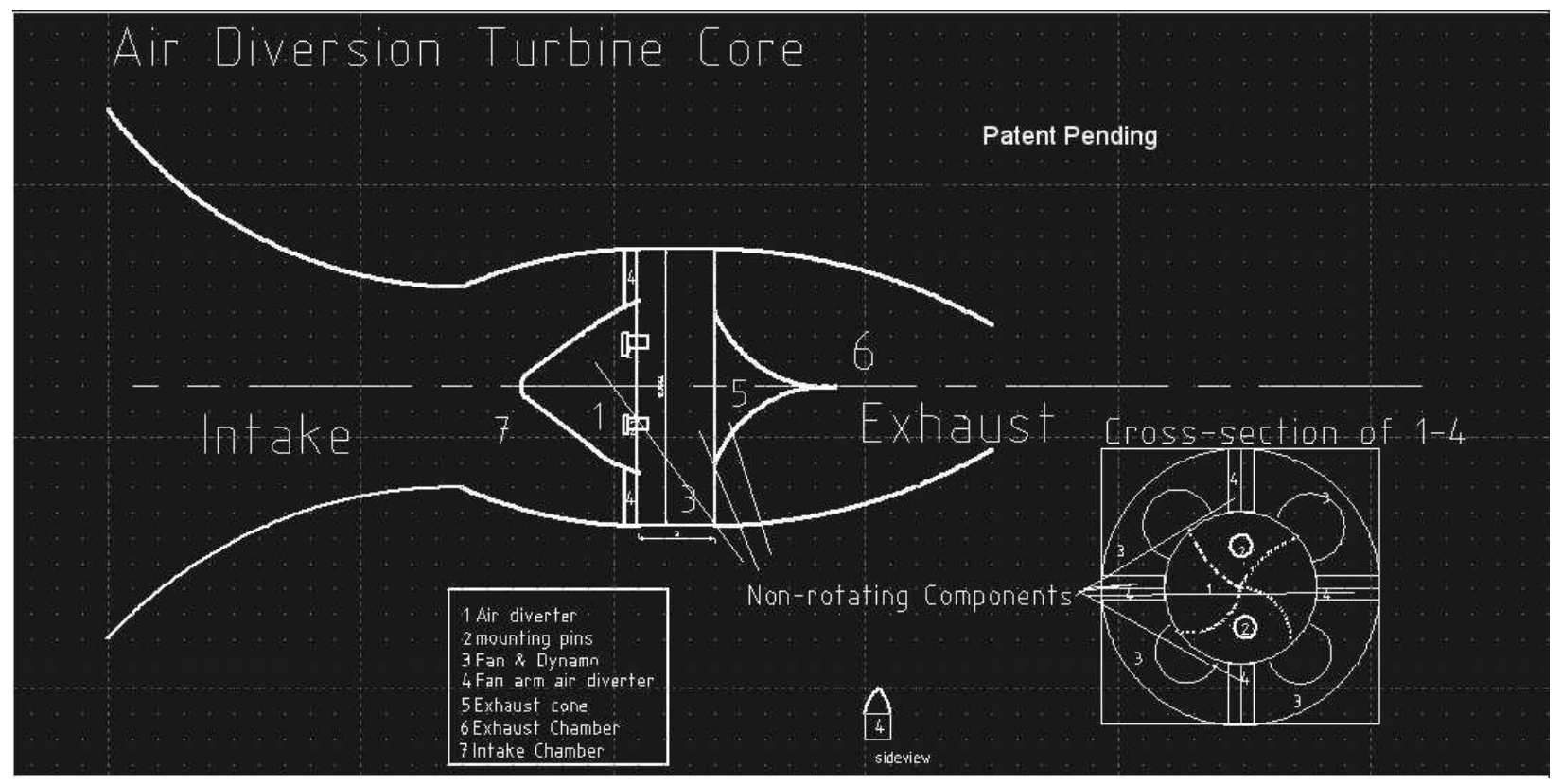

FIGURE 19. Original Turbine Concept from WEST Wind Power Inc.

Some parts of the inlet and exit geometry were tested jointly as part of a separate project, and some results of both are presented here to completely describe the evolution of the overall design (Russ, 2011). The first item addressed was finding an optimized intake geometry. Arbitrary specifications were tested at first, based on the intention for 
residential use. Therefore, the device should have a footprint of about a square meter. With this in mind, the radius of the throat was set equal to twenty-five centimeters, the intake equal to a meter, and the total length equal to 1.0 to 1.5 meters. The actual parabolic intake cone would measure seventy-five centimeters, with the throat extending another thirty centimeters. Lastly, the material from which the entire device was crafted was to be uniformly 2.5 centimeters thick. The design at this stage resembled a trumpet with an overly large bell. The next step in the process was to create a number of curves representing the shape of the intake to test, ranging from a parabola to a hyperbola with each end terminating at either the one meter radius or on the quarter-meter throat.

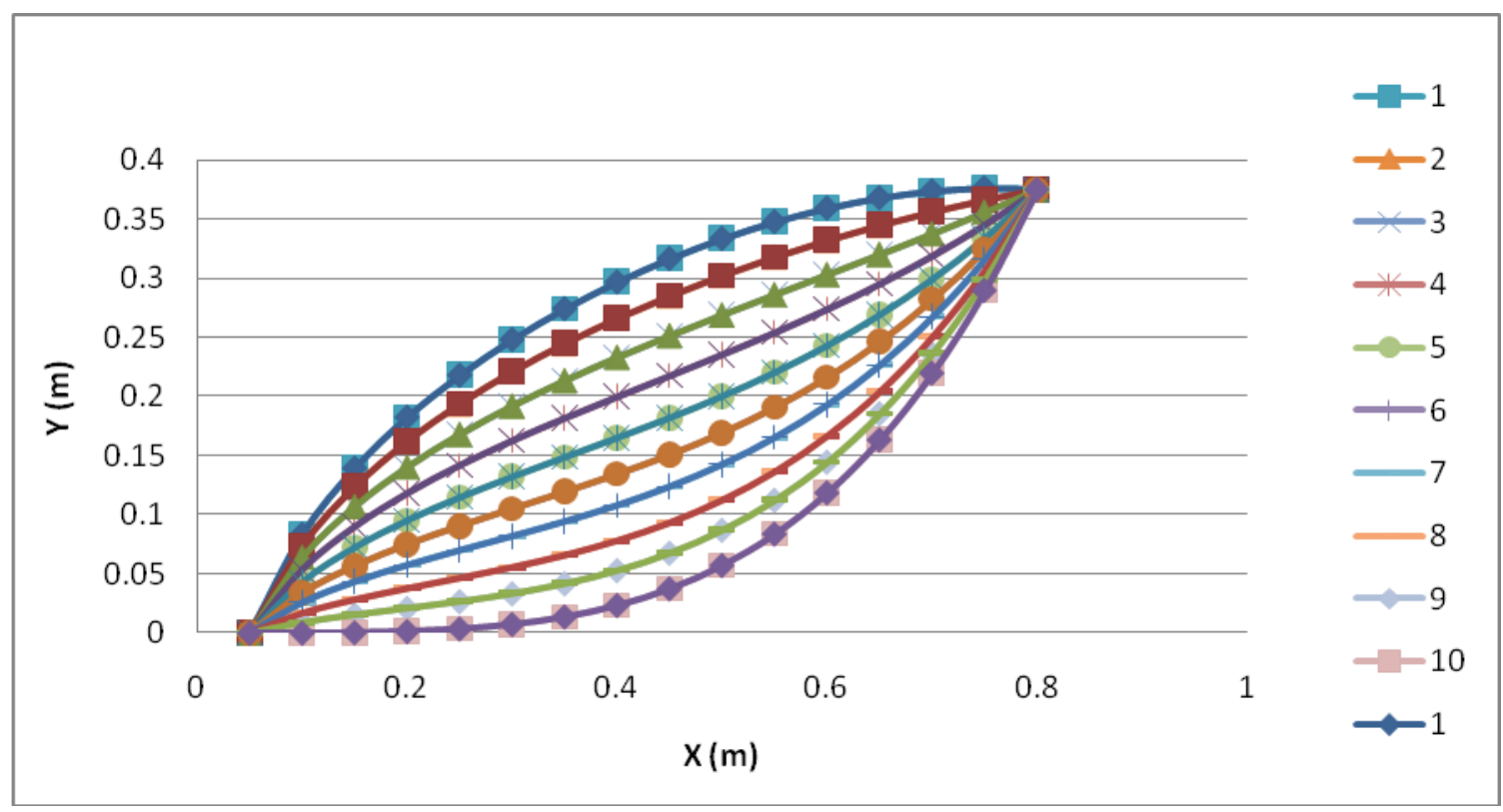

FIGURE 20. Curves Representing Inlet Shapes. The Outer Edge of the Cone Inlet is at $0.5 \mathrm{~m}$

The curves were controlled by two factors. The first was an arbitrary measure of concavity, $\mathrm{C}_{\mathrm{c}}$, ranging from -0.18 to 1 . The curves displayed in Figure 20 were described with the equation: 


$$
\mathrm{Y}=\mathrm{C}_{\mathrm{s}} *\left(\left(\mathrm{C}_{\mathrm{c}}\right) \mathrm{X}^{4}+\left(1-\mathrm{C}_{\mathrm{c}}\right) \mathrm{X}^{1 / 4}\right)
$$

As $C_{c}$ approached 1, the shape approached a parabola described by $X^{4}$. As $C_{c}$ approached 0 , the shape approached a hyperbola described by $\mathrm{X}^{1 / 4} \cdot \mathrm{C}_{\mathrm{c}}$ was rendered to less than 0 to allow for testing of a wider variety of curves, from a horizontal tangent at the junction between the inlet and throat, to a nearly vertical tangent. The second constant, $\mathrm{C}_{\mathrm{s}}$, was a scaling factor, which fit the curve into the desired range: rising 0.375 meters over a 0.75 meter run.

Fluent ran models with the inlet curves from Figure 20 with a laminar model (one using only equations 1, 4 and 6) and the results from these models in Table I show that there was a correlation between curvature and throat velocity.

TABLE I.

\section{THROAT VELOCITIES WITH VARYING GEOMETRIES}

\begin{tabular}{|l|l|}
\hline Curve & Throat V \\
\hline 1 & $6.5 \mathrm{~m} / \mathrm{s}$ \\
2 & $16.5 \mathrm{~m} / \mathrm{s}$ \\
3 & $3.3 \mathrm{~m} / \mathrm{s}$ \\
4 & $4.5 \mathrm{~m} / \mathrm{s}$ \\
5 & $11.3 \mathrm{~m} / \mathrm{s}$ \\
6 & $3 \mathrm{~m} / \mathrm{s}$ \\
\hline
\end{tabular}

There are two peaks in the data, and for curves more convex than the sixth, the velocity stays relatively low, around $5 \mathrm{~m} / \mathrm{s}$. As shown in Figure 21, a parabolic curve will create destructive eddies in the throat of the device. 


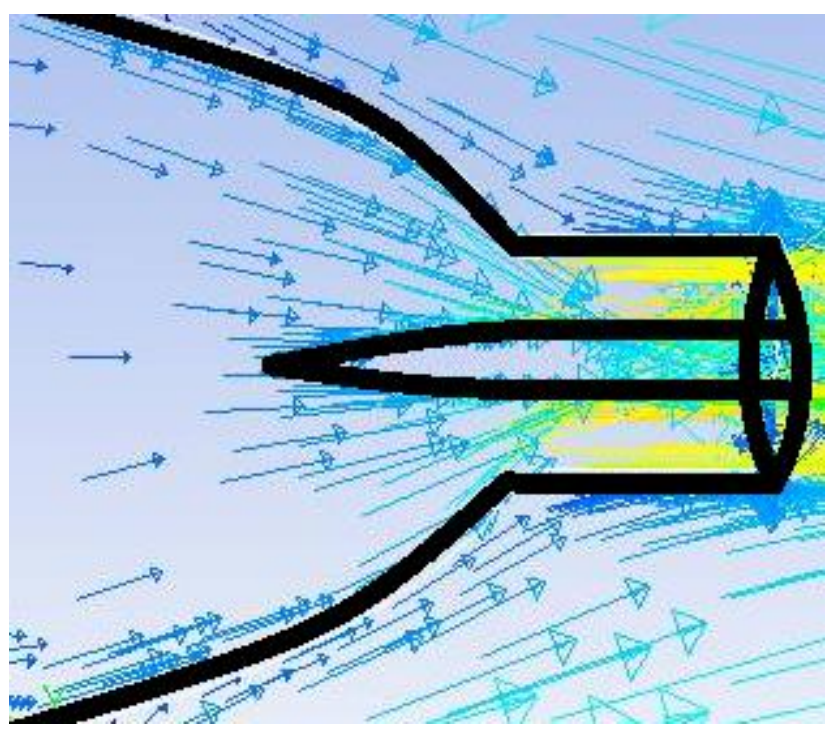

FIGURE 21. Vectors Collide with Center Cone in Front of the Throat. Longer, Hot Colored Arrows Represent Larger Velocities.

As a result, more parabolic curves (curves beyond number five) do not produce good results. These first models gave rather inflated velocities for the designs as discussed in the earlier section concerning mathematical models. The laminar mathematical algorithm used does not accurately reflect the air flow under these conditions. Although inaccurate, this data provided a basis for later models using the realizable k-€ turbulence model.

These models were run with the new equation set (Russ, 2011) and it was concluded that the third or fourth curve was the best designs for the inlet. These curves showed an acceleration of $15 \%$, from the ambient flow of $2 \mathrm{~m} / \mathrm{s}$ to $2.3 \mathrm{~m} / \mathrm{s}$ in the device throat, with a turbulent model

At this point, the use of a cone to serve as a flow straightener became the focus of optimization. A cone may also be used to provide a structure from which fan blades may be mounted. After the best inlet curve shape was determined, other geometrical aspects of the device were considered that could improve the maximum velocity. An outlet 
shroud extending fifteen centimeters was added, its concavity mirroring that of the inlet curve (as described in the literature review, an outlet shroud is a proven method for use in wind concentration (Hau, 2006)). Also, as shown in Figure 22, "club" shaped diverters were added at the end of the inlet curves to alter the incoming turbulent kinetic energy. This reduced the effects of energy reducing eddies within the inlet.

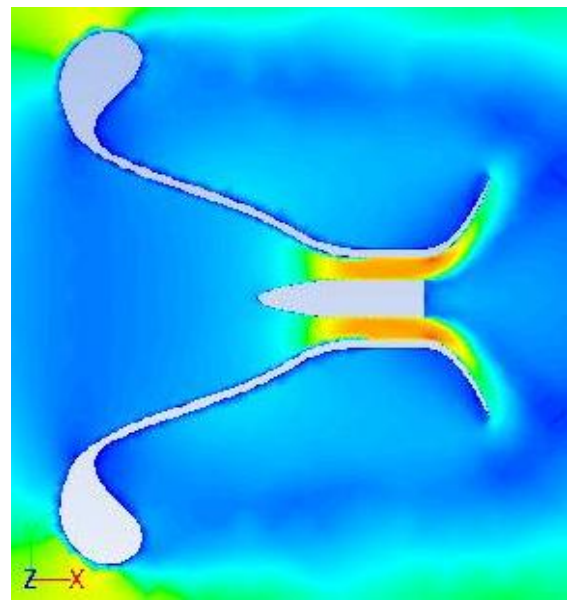

\section{FIGURE 22. New Turbine Design with Cone}

This design had a throat velocity of $2.6 \mathrm{~m} / \mathrm{s}$. Within this new turbine template, the shape of the cone was altered by adjusting two parameters: the length and diameter. The goal was to determine a correlation between the length/diameter in order to determine a final cone design.

The cone was tested for lengths of 100, 60, 40 and 20 centimeters with a cone diameter of seven centimeters. Rather than a true cone, the shape consisted of a hyperbolically curved nose attached to a cylinder. The parabola was described by the equation $\mathrm{Y}=\mathrm{X}^{2}$, and was scaled to and connected with a 30 centimeter long cylinder with a diameter of 7 centimeters. The lengths listed above describe the length of the hyperbolic section of the cone. Figure 23 shows the geometries of the four cones tested, 
while Table 2 shows the resulting maximum velocities at the throat. All velocity contours in this thesis have units in $\mathrm{m} / \mathrm{s}$.

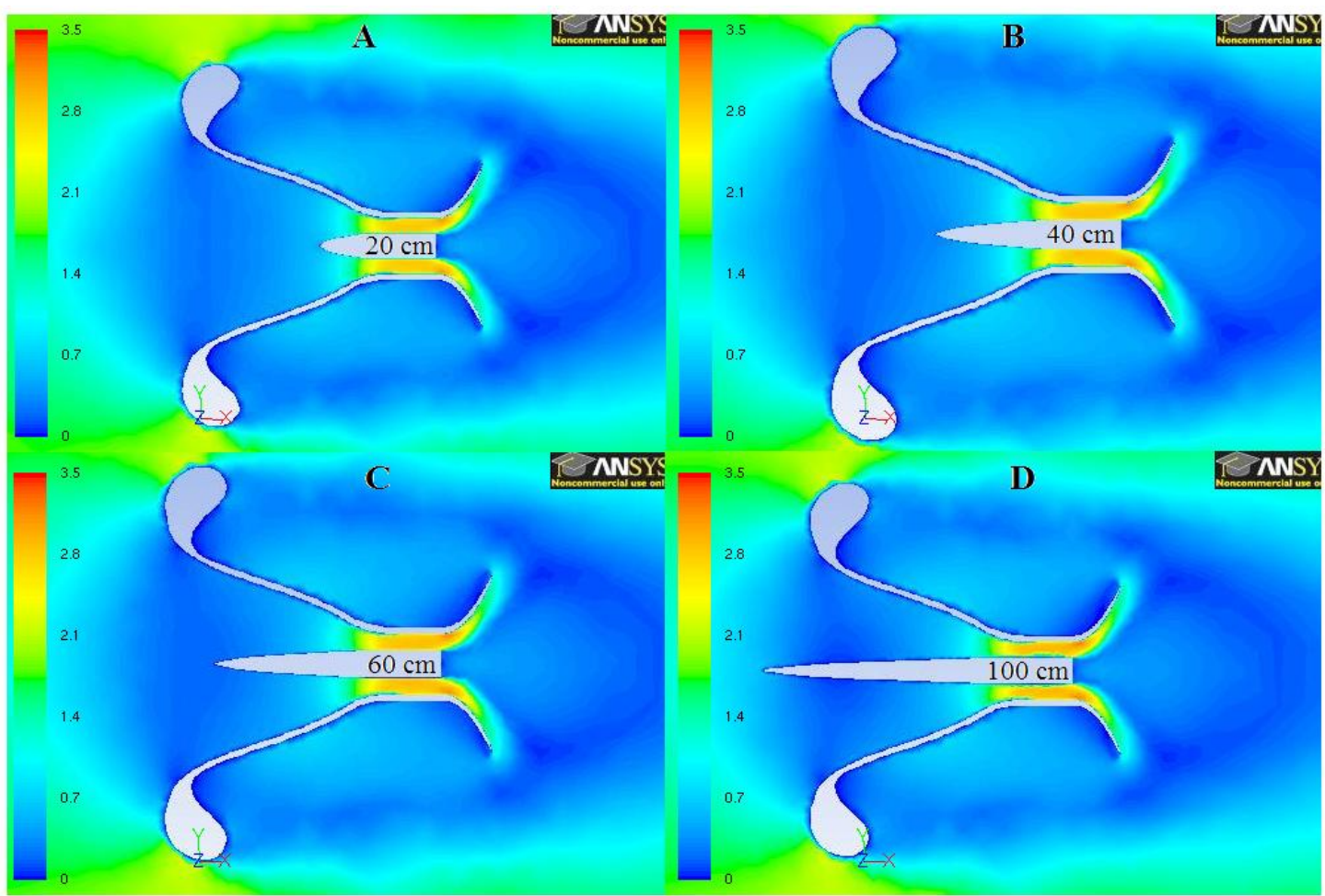

FIGURE 23. Velocity Contours for Cone Lengths of 20, 40, 60, and $100 \mathrm{~cm}$

TABLE II.

\section{THROAT VELOCITIES WITH DIFFERING LENGTHS}

\begin{tabular}{|l|llll|}
\hline Length $(\mathrm{cm})$ & 20 & 40 & 60 & 100 \\
\hline Throat Velocity $(\mathrm{m} / \mathrm{s})$ & 2.75 & 2.8 & 2.85 & 2.9 \\
\hline
\end{tabular}

It is shown in Table II above that the throat velocity increases with the length of the cone. This is likely because the cone served to rectify the inflowing air, reducing the appearance and intensity of eddies in the inlet, allowing the influx to behave less turbulent and more like a laminar system. With a longer cone, these effects are more pronounced. Although an even longer cone may a slightly better effect, a cone with a 
length beyond 1 meter would push the footprint of the turbine out of the range of the intended application. Additionally the cone will have little effect on the air around it once beyond the inlet as the data from Table II shows that the potential gain decreases as the cone lengthens. From this data, it appears that a length of 100 centimeters is the optimized cone length for this project.

The second parameter to be optimized was the cone diameter. There are four options considered here. The diameter of the cone is limited by the throat diameter, which is 25 centimeters. Additionally, the diameter cannot be smaller than two centimeters due to structural stability concerns. Therefore, the four diameters tested were $2,8,14$, and 20. The shapes and throat velocities for these designs are shown in Figure 24 and Table III respectively:

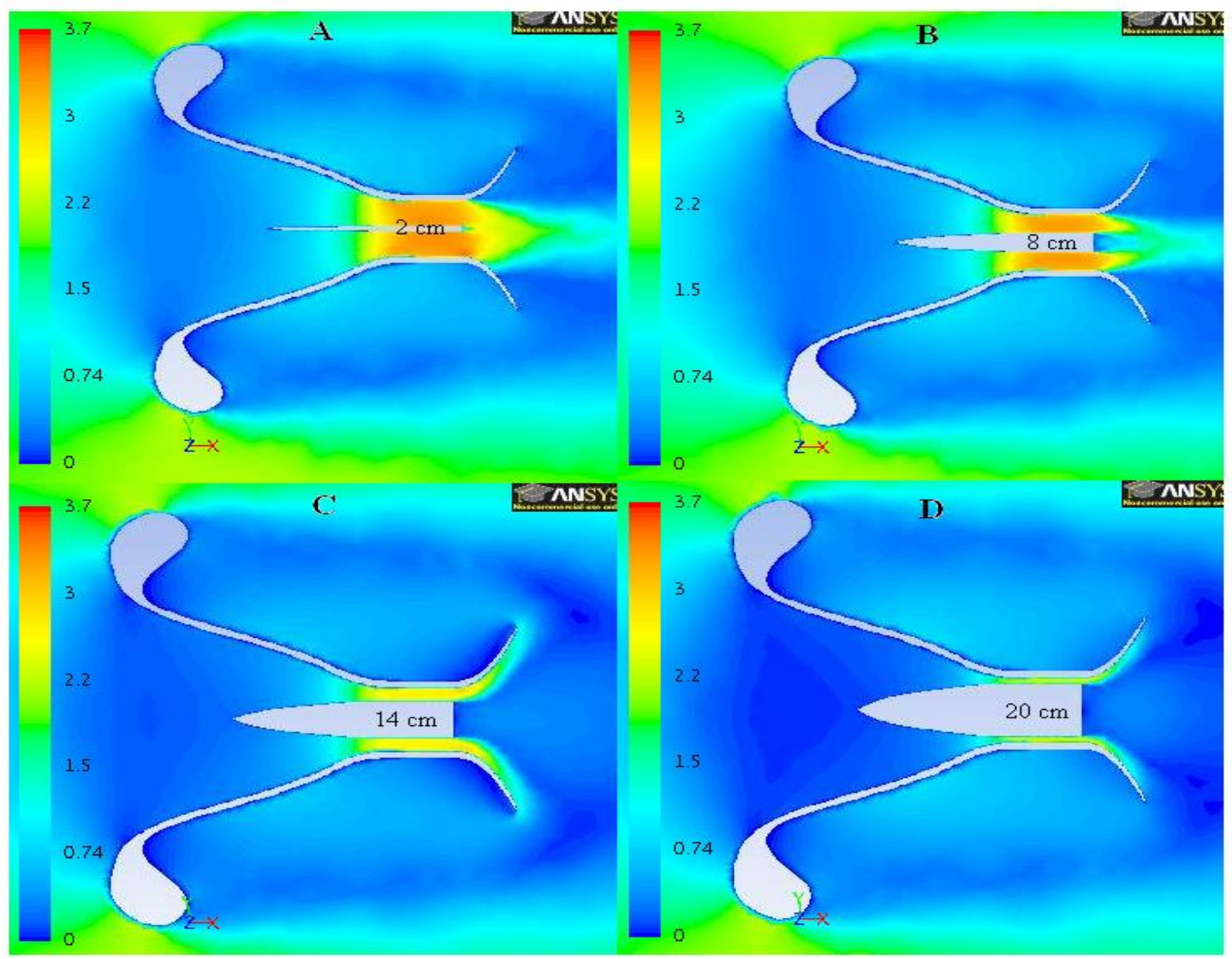

FIGURE 24. Velocity Contours for Cone Diameters of 2, 8, 14, and $20 \mathrm{~cm}$ 
TABLE III.

THROAT VELOCITIES WITH VARYING CONE DIAMETERS

\begin{tabular}{|l|llll|}
\hline Radius $(\mathrm{cm})$ & 2 & 8 & 14 & 20 \\
\hline Throat Velocity $(\mathrm{m} / \mathrm{s})$ & 3.25 & 3.2 & 2.6 & 2.34 \\
\hline
\end{tabular}

As shown in Table III, the thinnest cone produces the fastest velocity. Assuming the effects are cumulative, a long, thin (100 cm length and $2 \mathrm{~cm}$ diameter) cone should best concentrate wind velocity. The turbulent kinetic energy and velocity profile of a design incorporating this preferred cone geometry is shown below in Figures 25 and 26. Note that the velocity is greater than the maximum of either a long cone or a thin cone alone, as shown in Tables II and III. All turbulent kinetic energy profiles in this thesis will be in units of $\mathrm{m}^{2} / \mathrm{s}^{2}$. 


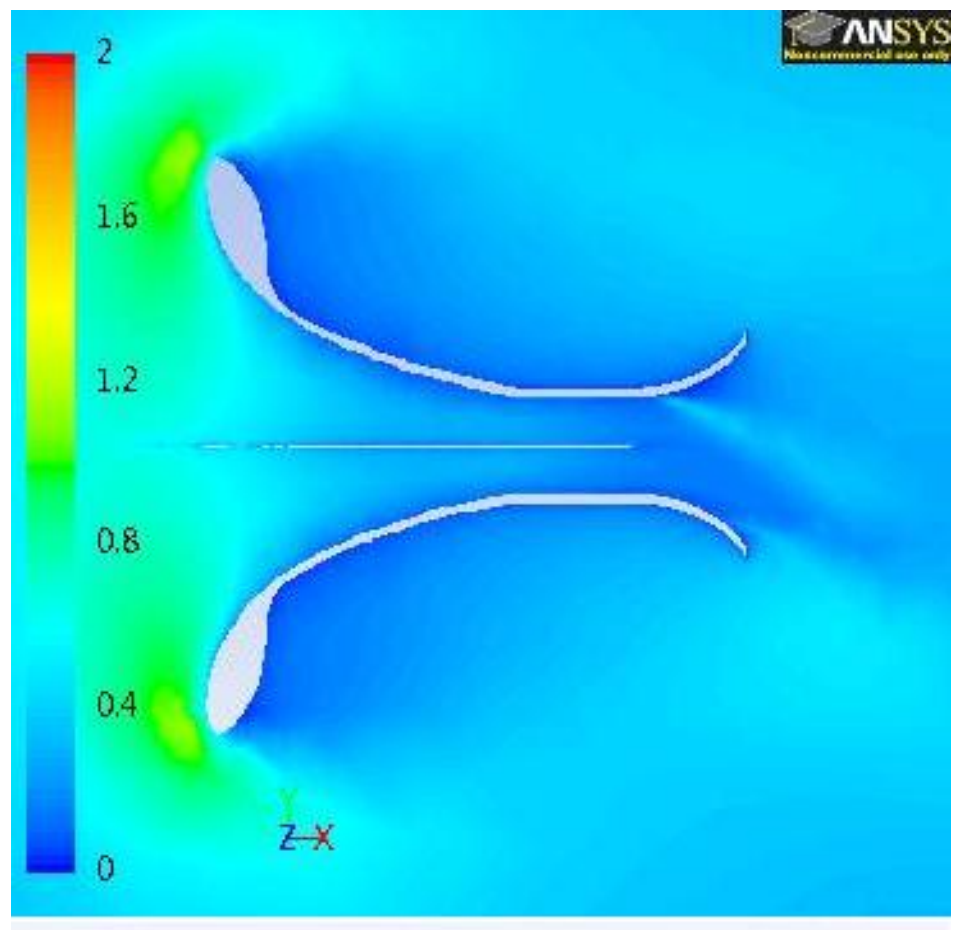

Contours of Turbulent Kinetic Energy $(\mathrm{k})(\mathrm{m} 2 / \mathrm{s} 2) \quad$ M... ANSYS FLUENT 12.1 ( $3 \mathrm{~d}$, pbns, rke)

FIGURE 25. Turbulent Kinetic Energy Profile of Preferred Cone Geometry

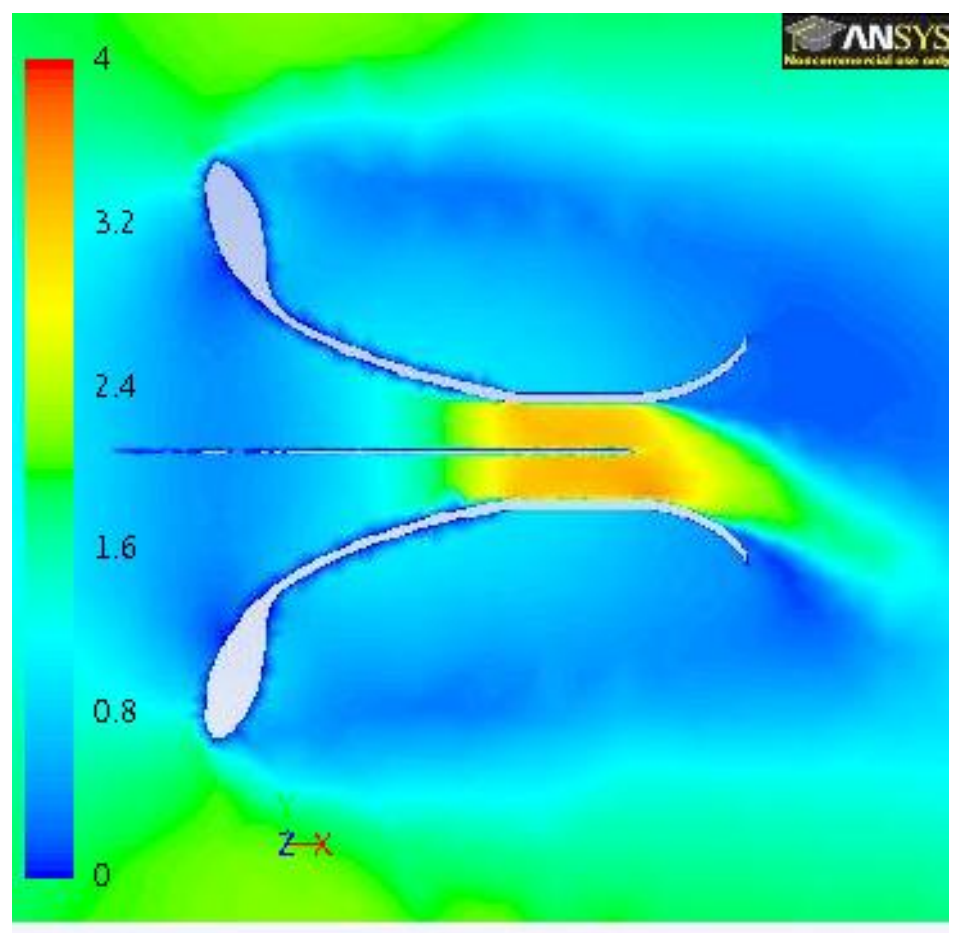

Contours of Velocity Magnitude (m/s) Mar 16, 2011 ANSYS FLUENT 12.1 (3d, pbns, rke)

FIGURE 26. Velocity Profile of Preferred Cone Geometry 
As shown in Figure 25, the turbulence inside the cone is approximately equal to that of the ambient air outside the cone. Additionally, the cone disrupts the area of high turbulence around the inlet opening, allowing more air into the turbine. As shown in Figure 26, the maximum throat velocity is about $3.3 \mathrm{~m} / \mathrm{s}$, showing that the effects of diameter and length are cumulative, with the effect of the thin cone dominating that of length. This is expected, as the variance in velocities between the shortest and longest cone is only about $0.15 \mathrm{~m} / \mathrm{s}$, while the difference for the diameter is almost $1 \mathrm{~m} / \mathrm{s}$. The $3.25 \mathrm{~m} / \mathrm{s}$ velocity shown in Table III, from the $2 \mathrm{~cm}$ diameter cone, gains as much speed as would be expected from Table II for the length change, increasing the maximum throat velocity to $3.3 \mathrm{~m} / \mathrm{s}$.

The next concern was to reduce the turbulence seen at the inlet of the device. Looking at Figure 25, even with the aid of the flared rim, back-pressure from the throat was a serious limiting factor to the total throughput of the turbine. To attempt to alleviate this backpressure and reduce the turbulent "wall" at the inlet, several designs were tested, which includes the addition of various vortex breakers and pressure relief slits. Their designs will be discussed in detail in the next section. Figure 27 shows the eight designs which were tested: 


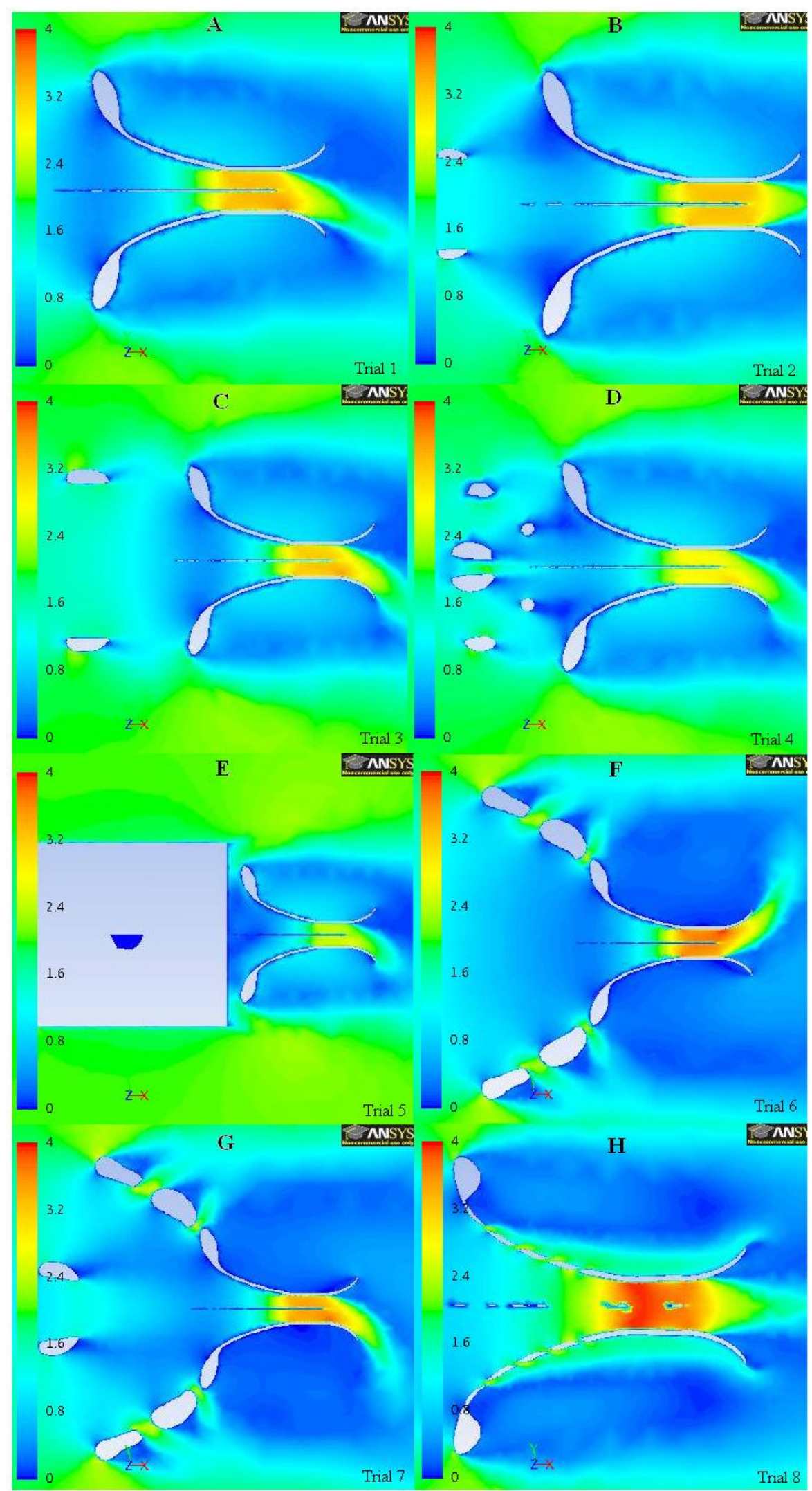

FIGURE 27. Velocity Profiles of Slit and Vortex Breaker Designs 


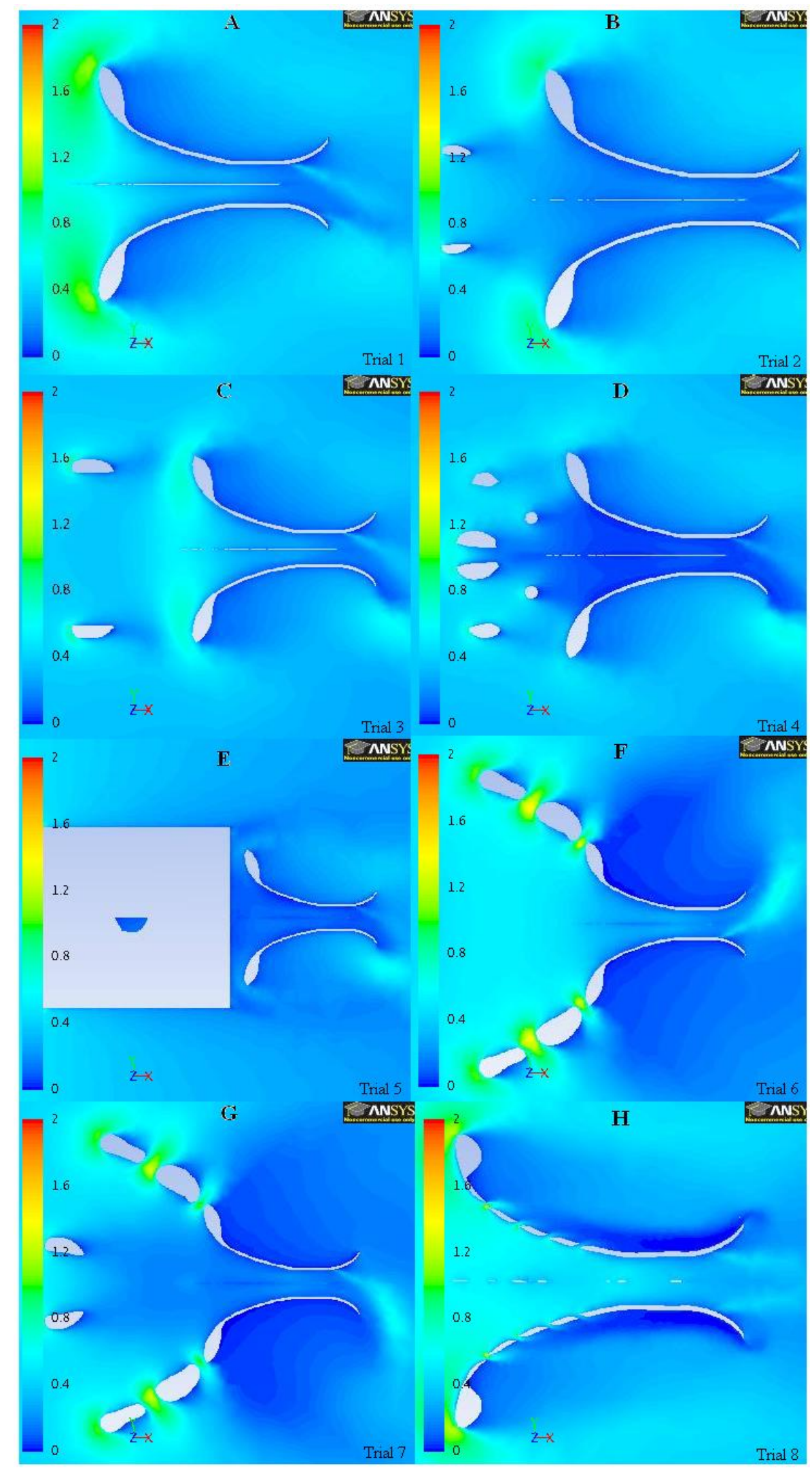

FIGURE 28. Turbulence Profiles of Slit and Vortex Breaker Designs 
Of the eight trials, the first was a control. It incorporates the results of the cone trials, as shown in Figure 26. The maximum throat velocity for the control was $3.3 \mathrm{~m} / \mathrm{s}$; this is the benchmark against which the other designs will be tested. The rest are numbered in the order in which they were tested, as each trial contributed some knowledge to the next. The images shown are radial cross sections; therefore, the two oblong shapes in front of the funnel are actually part of one ring. The first trial was an attempt to "trip the boundary layer," a phrase meaning to create eddies in a stream so the flow will already be turbulent when it reaches an obstacle. This works best in the case of water or other liquids, where the initial flow is laminar (McCabe et al., 2000). In this case, only a slight speed decrease was seen, to $3.28 \mathrm{~m} / \mathrm{s}$. The ring served to rectify the flow into the funnel, and shield the funnel slightly from turbulence, shown in Figure 28$\mathrm{C}$, but affected little other change.

Trial 2 attempted to use a ring with a larger diameter to produce a quicker flow and reduce turbulence. In this scenario, the ring shielded the funnel's lip from some turbulence, reducing the average turbulence by about $10 \%$. However, velocity was not positively improved, as the model only produced a maximum velocity of $3.25 \mathrm{~m} / \mathrm{s}$. The third trial consisted of several rings thrown together in an attempt to see if the turbulence reduction in Trials 1 and 2 were cumulative, and if by reducing turbulence a velocity increase could be affected. From Figures 27-D and 28-D, it is shown that the effects are more than cumulative. There is comparatively little turbulence in the cone throat compared to any other model. However, the velocity dropped further to $2.8 \mathrm{~m} / \mathrm{s}$, showing that the use of obstructions to reduce turbulence will also reduce the amount of air flowing through the device. 


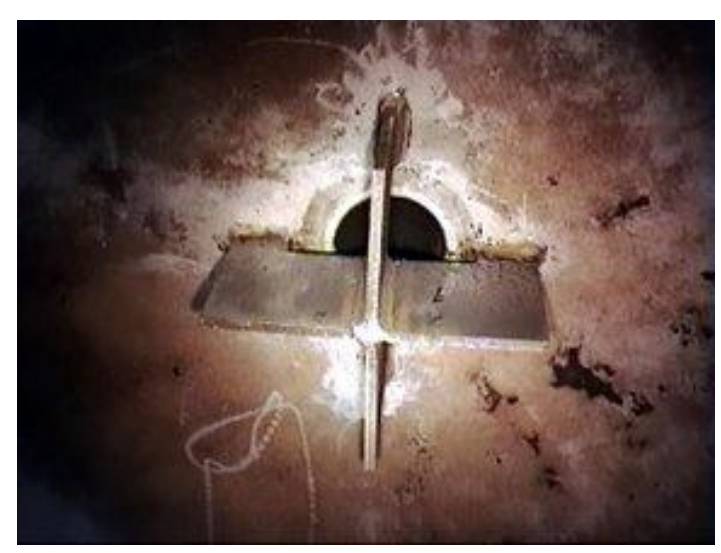

FIGURE 29. Vortex Breaker (Processing, 2008)

Trial 4 was a final attempt to break vortexing. Instead of using simple rings, however, a conventionally cross-shaped vortex breaker was used. The type used was similar to those found in refinery tanks to prevent cavitations in tank bottoms pumps (see Figure 29). It worked as was expected; turbulence was greatly reduced across the board, but so was velocity as shown in Figures $27-\mathrm{E}$ and $28-\mathrm{E}$, where velocity was $2.4 \mathrm{~m} / \mathrm{s}$, and turbulence is reduced ten-fold from the control. This data supported the conclusions from Trial 3. Therefore, from the first four trials, it was concluded that rings or vortex breakers were ineffective for concentrating wind velocity.

Trials 5 and 6 were performed to see what would happen if rings were used to extend the size of the funnel, and how a central ring would interact with such an array. These designs were the only ones, up to this point, to produce an actual velocity increase, with trials 5 and 6 resulting in a velocity of $3.6 \mathrm{~m} / \mathrm{s}$ and $3.45 \mathrm{~m} / \mathrm{s}$. It should be noted that in this case even one ring decreased the velocity noticeably. These results were expected in that a bigger cone should result in a faster velocity. However, what was unexpected and extremely fortuitous is the revelation that the space between the rings was so turbulent there was little flow through them, forming a sort of wall while still relieving 
backpressure. This was a significant breakthrough because the addition of slits to the control design increased the velocity to $3.8 \mathrm{~m} / \mathrm{s}$, the highest velocity yet. This design still fit in the intended footprint, and increased wind speed by another $30 \%$.

After discovering that pressure-relief slits would improve wind speed concentration, several other designs were tested (Figure 30).

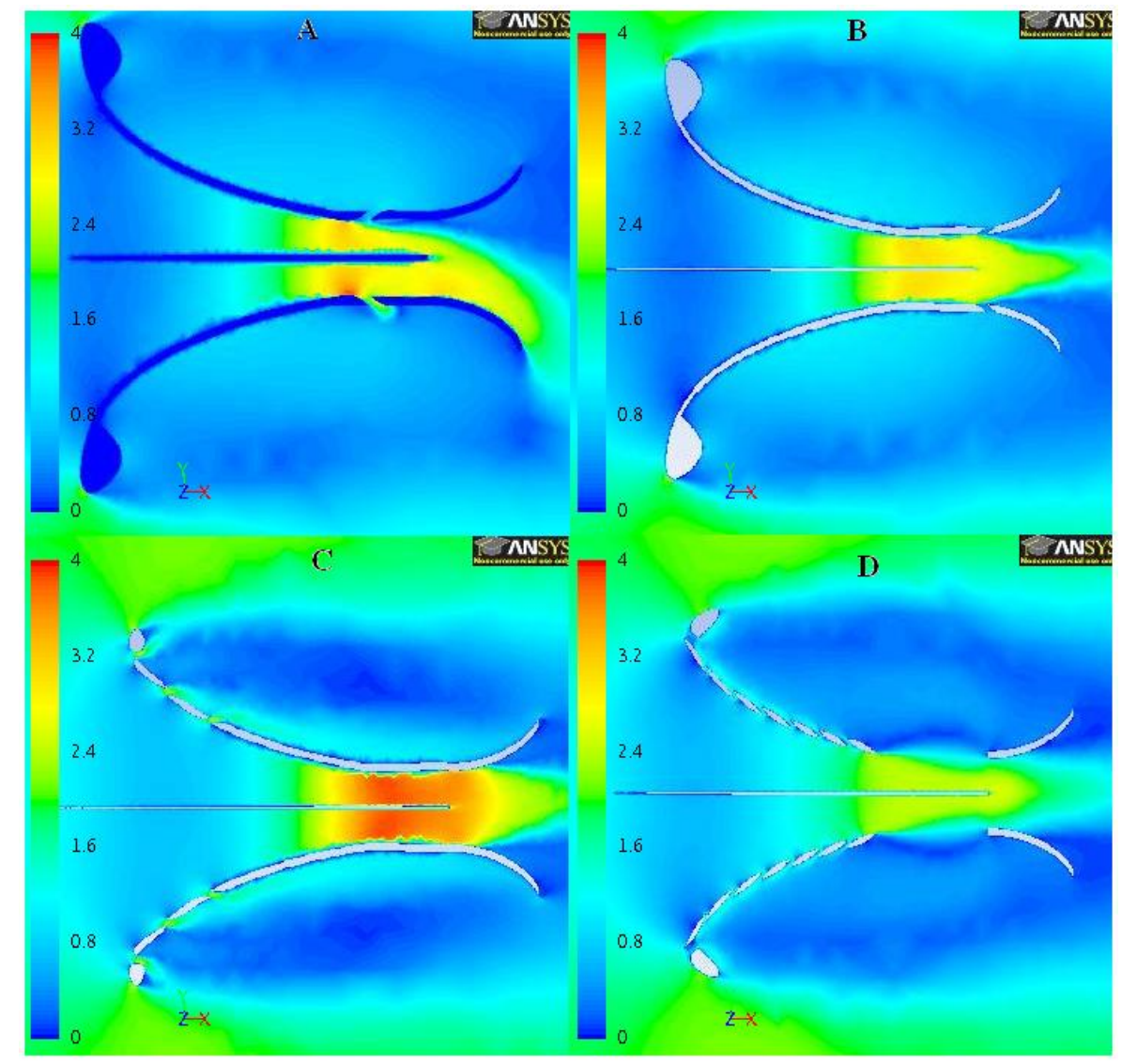

FIGURE 30. Slit Designs

However, unlike the slit design in Figure 28-H, only Design 3 showed improvement over the control (Trial 7). Designs 1 and 2, shown in Figure 30-A and B, featured a slit in the middle and rear sections of the apparatus; they both performed relatively poorly; a slit in the middle increased velocity slightly, to $3.4 \mathrm{~m} / \mathrm{s}$ compared to the $3.3 \mathrm{~m} / \mathrm{s}$ control. A slit 
in the rear section actually reduced the throat velocity, down to $3.0 \mathrm{~m} / \mathrm{s}$. This is likely because the purpose of a rear shroud is to create a pressure differential. Slits in this location reduced its capacity to perform this function, reducing the velocity. A slit in the throat helped relieve some of the backpressure in the apparatus, but, as most of the backpressure was generated near the rim of the windward cone, this design was less effective. The third design had one fewer slit than Trial 7 (Figure 28-H). Here, the slits were focused near the rim of the cone, with only a slight decrease down to $3.77 \mathrm{~m} / \mathrm{s}$ in velocity, compared to $3.8 \mathrm{~m} / \mathrm{s}$ in Trial 7. This shows that most backpressure was caused by the cone, and the greatest need for slits was in the front. In other locations, a slit would do more harm than good. The important factor was the existence of front slits, and not necessarily how many. Lastly, Trial 4 (Figure 30-D) tested how wind would flow without a throat wall if slits were used. Despite minimal housing, this design increased wind speed by $20 \%$. However, this increase is overshadowed by the $90 \%$ increase seen by Trial 3 (Figure 30-C).

The final iteration of testing focused on optimizing the slit design further, as well as the cone rim shape. The cone rim exhibited the largest amount of turbulence in the designs; the question was raised of whether this could be changed, or even removed entirely. The designs shown in Figure 31 use a design created by another research student from Trial 3 in Figure 30-C (Russ, 2011). This design increased the leeward shroud length by a factor of two, increasing the maximum velocity to $4.55 \mathrm{~m} / \mathrm{s}$ and is shown in Figure 31. This design is used as the control design for the trials shown in Figure 32. 


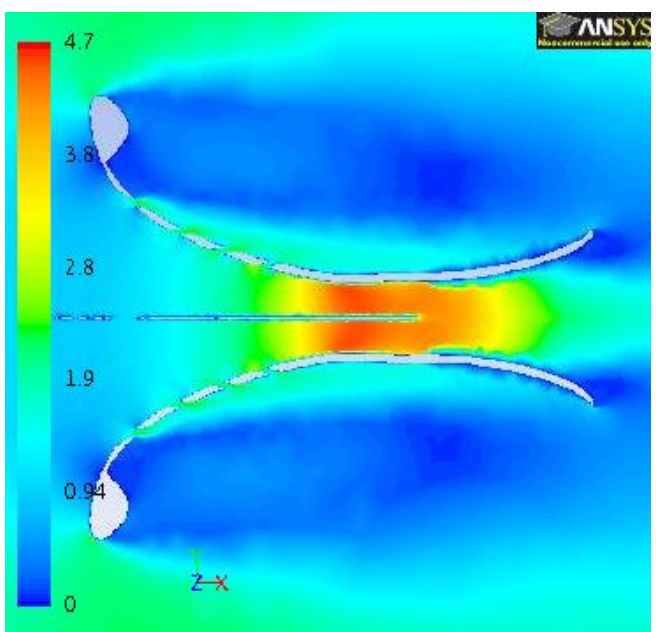

FIGURE 31. Control Design for Slit Optimization 


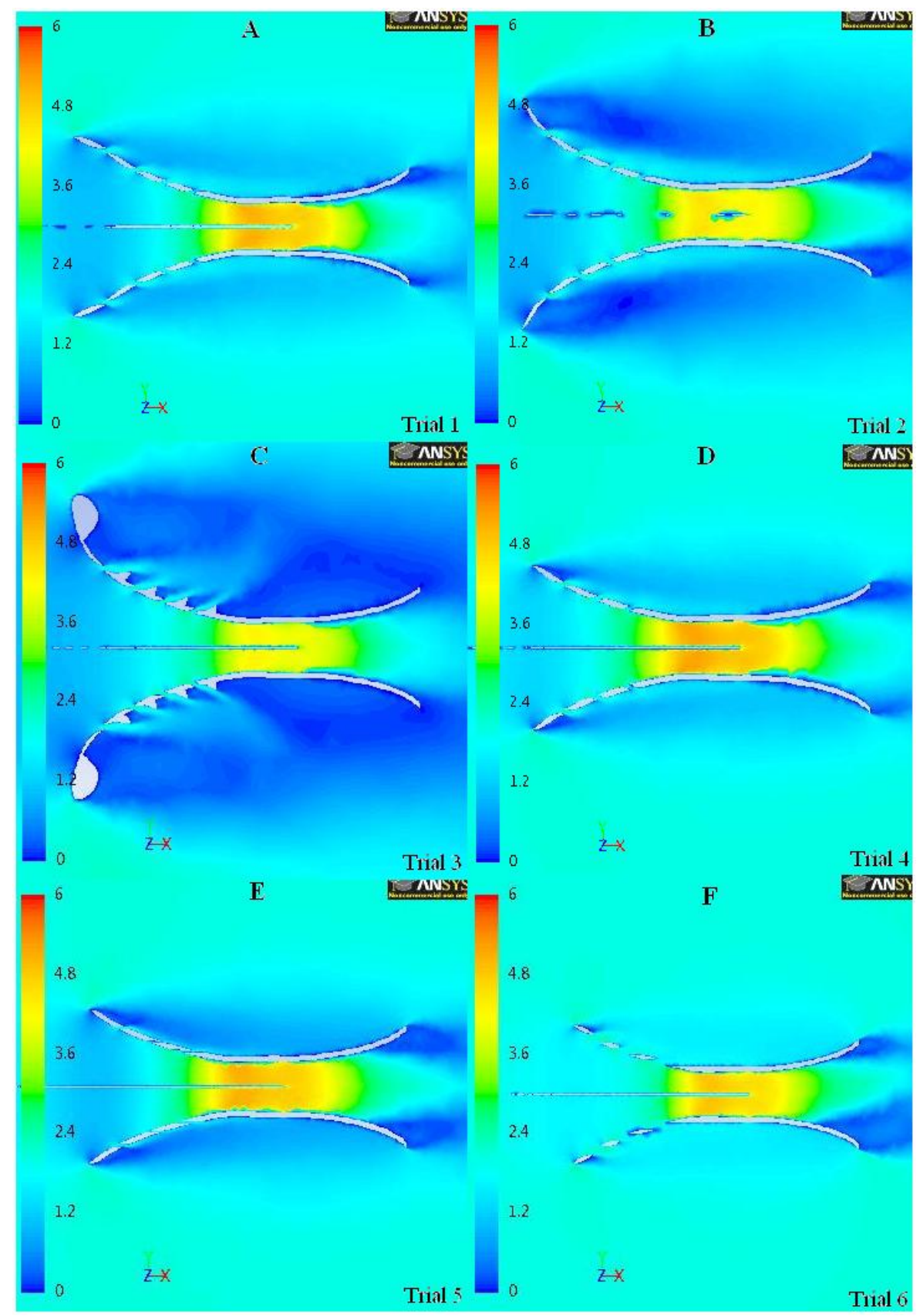

FIGURE 32. Slit Designs 2

Trials 1, 2 and 4 (Figure 32-A, B, and D) focus on the "club" shape on the rim of the cone. In Figure 32-B, the "club" segment is removed from the most windward ring. In number Figure 32-A, the "club" is still removed, but the remaining rim is bent to be 
parallel to incoming stream lines to reduce turbulence. In Figure 32-D, the most windward segment is removed alltogether. Compared to the control velocity of $4.55 \mathrm{~m} / \mathrm{s}$ from Figure 31, Trials 1 and 2 showed improvement, while Trial 2 stayed approximately the same, having a similar hydrolic profile. Trial 1 increased the velocity to $5.07 \mathrm{~m} / \mathrm{s}$, while Trial 4 showed the largest increase, at $5.17 \mathrm{~m} / \mathrm{s}$. The "club" section was, therefore, shown to be entirely unnessessary, and actually detremental to wind concentration. Additionally, with a smaller inlet radius, the concentration efficiency actually increased greatly, from about $13 \%$ in the control to about $73 \%$.

Trials 3, 5 and 6 (Figure 32-C, E, F) are all tests to determine if changing the shape of the slits would increase the velocity. In Trial 3, shrouds are added to determine whether this would increase throat velocity. It had little effect, slightly decreasing the throat velocity to $4.3 \mathrm{~m} / \mathrm{s}$. Trials 5 and 6 were designed from the geometry of Trial 4 , and tested the effect of the slit width on the throat velocity. These two should be compared to Trial 4, as it was the template model. In Trial 5, the slit width was reduced by half. There was little effect, with a slight reduction in velocity to $5.07 \mathrm{~m} / \mathrm{s}$ compared to 5.17 $\mathrm{m} / \mathrm{s}$, the velocity in Trial 4. In number 6 , the slit width was doubled, resulting in a significant reduction in throat velocity relative to Trial 4 , to $4.87 \mathrm{~m} / \mathrm{s}$.

Results show that the slit width in Trial 4 is best, in which the the pressure relief benefits most exceed the loss of throughput in the system through the slits. Number 4 was chosen as the final design for the system, capable of accelerating a mass of air by a factor of over $250 \%$. Final blueprints for the best designs can be found in Appendix A.

The prototype built from these blueprints performed admirably, producing acceleratiosn within $8 \%$ of the expected values from the Fluent models. This validates 
the use of the k- $\epsilon$ model as well as proving that an effective wind concentrator can be made with a windward funnel. Results from the prototype are shown below.

\section{Prototype Operation}

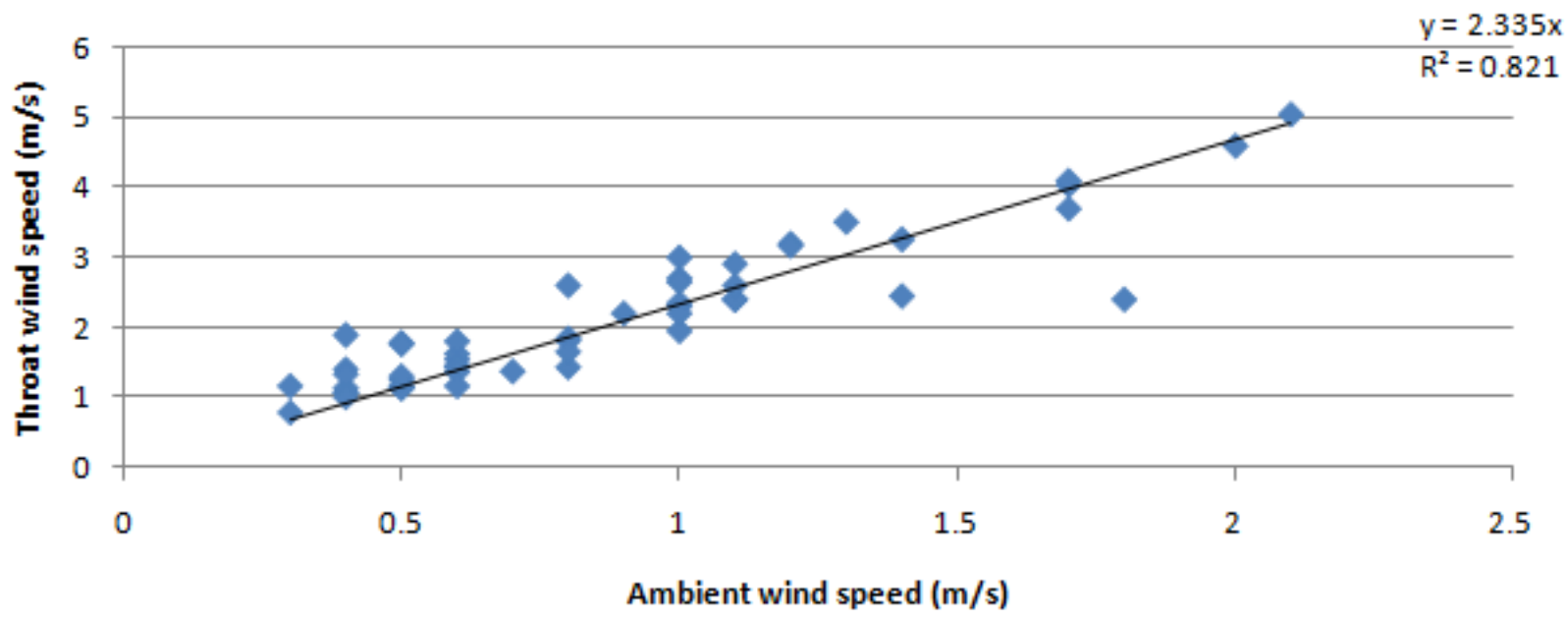

FIGURE 33. Experimental Data 


\section{Conclusions and Recommendations}

\section{A. Conclusions}

- A long thin cone is most effective at reducing turbulence and increasing velocity, improving wind speed by $10 \%$ more than any other tested design.

- "Vortex breaker" designs are ineffective at reducing turbulence in systems composed of low density fluids such as air.

- Pressure relief slits are effective at both reducing turbulence and producing a flow rectified with the concentrator geometry, increasing maximum velocity by $30 \%$.

- A properly designed forward concentrator can be used to increase the effectiveness of a wind concentrator.

- The final design transfers $74 \%$ of the momentum present at the entrance to the throat, with an overall increase in velocity of $256 \%$, from $2 \mathrm{~m} / \mathrm{s}$ to $5.17 \mathrm{~m} / \mathrm{s}$.

- Experimental data from the prototype performed within $8 \%$ of expectations, validating the CFD model used.

\section{B. Recommendations}

- Further optimization could be streamlined through the use of Linux coding to generate models of a certain type and perform "hands-off" optimizations of fine parameters such as slight width, concentrator diameter or concentrator profile.

- Investigate the effect of different types of turbine blades on power generation and effects of back-pressure.

- Re-optimize slit width and location with respect to different blade designs. 
- Re-optimize cone length and diameter with respect to the shortened inlet size.

- Optimize the size and shape of turbine and dynamo housings used to support and generate power from the array.

- Devise and implement a way to regulate wind speed within the throat relative to the external speed to improve efficiency and reduce the chances of catastrophic failure due to exceptionally high wind conditions. 


\section{References}

1. Chung, T. J. 2002. Computational Fluid Dynamics. Cambridge, UK: Cambridge University Press.

2. Ferziger et al, J. H. 2002. Computational Methods for Fluid Dynamics Third Edition. New York: Springer-Verlag.

3. J. M. Introductory Lectures in Turbulence Physics, Mathematics and Modeling. University of Kentucky, 2004. Print.

4. Lamox, Harvard, and Thomas H. Pulliam. 1999. Fundamentals of Computational Fluid Dynamics. NASA Ames Research Center.

5. ANSYS, Inc. 2009. FLUENT 12.0 Theory Guide. ANSYS.

6. McCabe, Warren, Julian Smith, and Peter Harriott. 2000. Unit Operations of Chemical Engineering. New York: McGraw-Hill.

7. Hau, Erich. 2006. Wind Turbines Fundamentals, Technologies, Applications and Economics Second Edition. Berlin: Springer-Verlag.

8. Davis, Edward L. 2010. US Patent 2010/0111689.

9. Becker, Bill. "Products," Aerotecture International Inc. July 10, 2010. Available from <http://www.aerotecture.com/products.html>. Accessed August, 2010.

10. Edwards, Victor R. 1996. US Patent 5,505,530.

11. Kane, Glen. 2010. US Patent 2010/0037541.

12. Accelerate Media. "WindTamer Turbines," WindTamer Corporation. Jun 10, 2010. Available from <http://www.windtamerturbines.com/windtamerturbines/>. Accessed August, 2010.

13. Heil, Clement et al. 2007. US Patent 7,218,011.

14. Loth, John L. 1977. US Patent 4,045,144.

15. Archer, Cristina L and Mark Z. Jacobson. 2005. "Evaluation of Global Wind Power." Stanford: Department of Civil and Environmental Engineering. 
16. "Vortex Breaker to Avoid Vapor Entrainment," Processing Magazine, July, 2008. Available from < http://webwormcpt.blogspot.com/2008/07/displayproblem-click-here-recommended.html>. Accessed November 2010.

17. Russ. David. 2011. "CFD Modeling of Entrance and Exit Geometries of Wind Speed Accelerators ." University of Louisville: Speed School of Engineering. 


\section{APPENDIX - A}

\section{Construction Specifications}

Attached are model drawings of the designed system. The testing was built around a $30 \mathrm{~cm}$ chamber, $25 \mathrm{~cm}$ in diameter, as housing for turbine blades. The devices should be fashioned from $2.5 \mathrm{~cm}$ thick plastic or comparable material. The following are interior points for the curve. Inlet and outlet funnels extend from here. The Table IV describes the shape of the model, from inlet to outlet:

TABLE IV.

WIND CONCENTRATOR PROFILE COORDINATES

\begin{tabular}{|l|l|l|l|}
\hline $\begin{array}{l}\text { Distance } \\
\text { from } \\
\text { opening }(\mathrm{m})\end{array}$ & $\begin{array}{l}\text { Diameter } \\
(\mathrm{m})\end{array}$ & $\begin{array}{l}\text { Distance } \\
\text { from } \\
\text { opening }(\mathrm{m})\end{array}$ & $\begin{array}{l}\text { Diameter } \\
(\mathrm{m})\end{array}$ \\
0.05 & 0.752 & 0.9 & 0.250 \\
0.1 & 0.676 & 0.95 & 0.250 \\
0.15 & 0.612 & 0.99 & 0.250 \\
0.2 & 0.558 & 1.03 & 0.251 \\
0.25 & 0.510 & 1.07 & 0.256 \\
0.3 & 0.468 & 1.11 & 0.263 \\
0.35 & 0.430 & 1.15 & 0.272 \\
0.4 & 0.395 & 1.19 & 0.283 \\
0.45 & 0.364 & 1.23 & 0.297 \\
0.5 & 0.336 & 1.27 & 0.313 \\
0.55 & 0.310 & 1.31 & 0.331 \\
0.6 & 0.288 & 1.35 & 0.352 \\
0.65 & 0.267 & 1.39 & 0.376 \\
0.7 & 0.250 & 1.43 & 0.404 \\
0.75 & 0.250 & 1.47 & 0.439 \\
0.8 & 0.250 & 1.51 & 0.483 \\
0.85 & 0.250 & 1.55 & 0.550 \\
\hline$h e n$ & 0.250 & & \\
\hline
\end{tabular}

Tolerance to the nearest $5 \mathrm{~mm}$ is reasonable. 


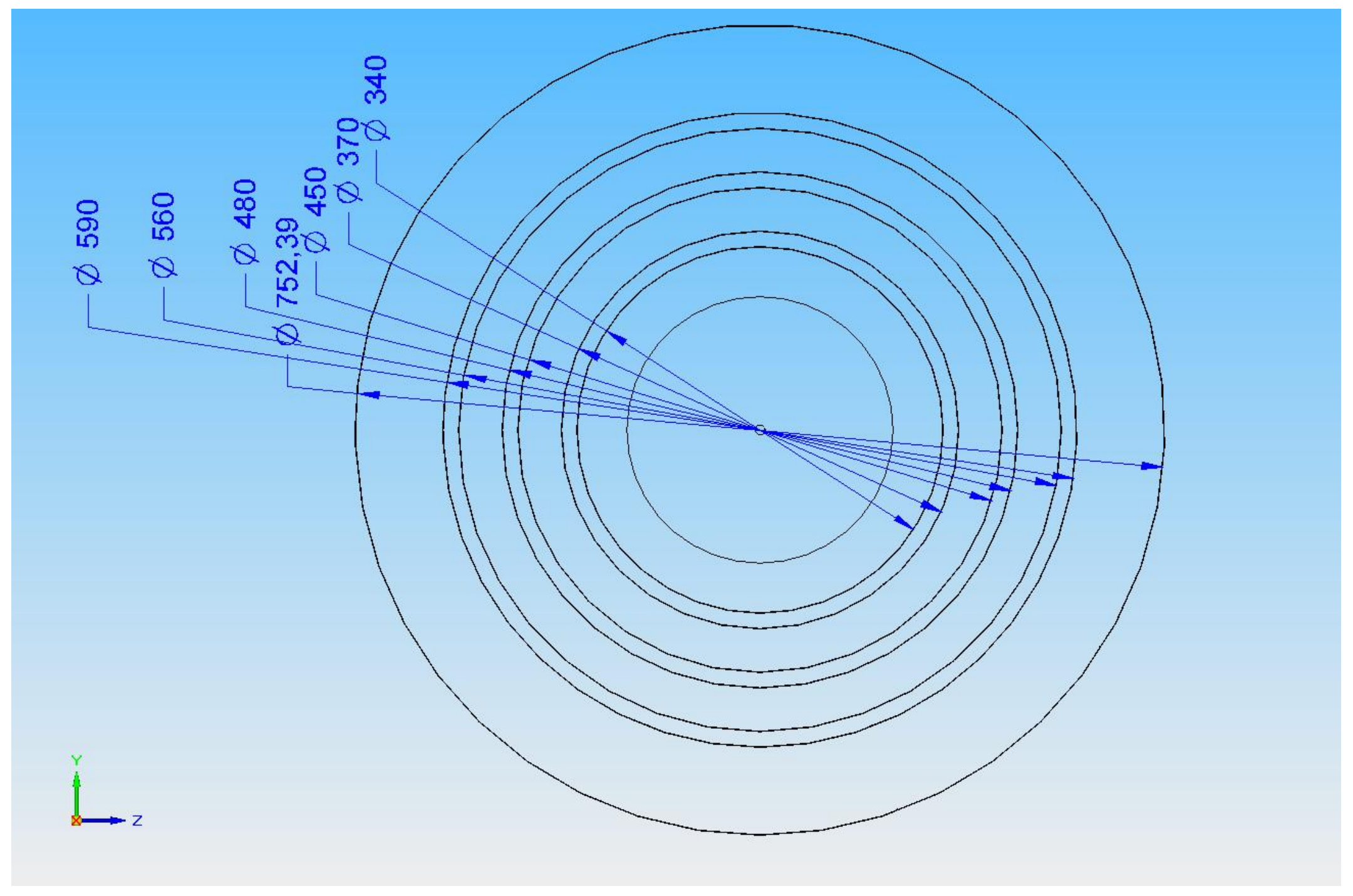

FIGURE 34. Front View (All Units in mm) 


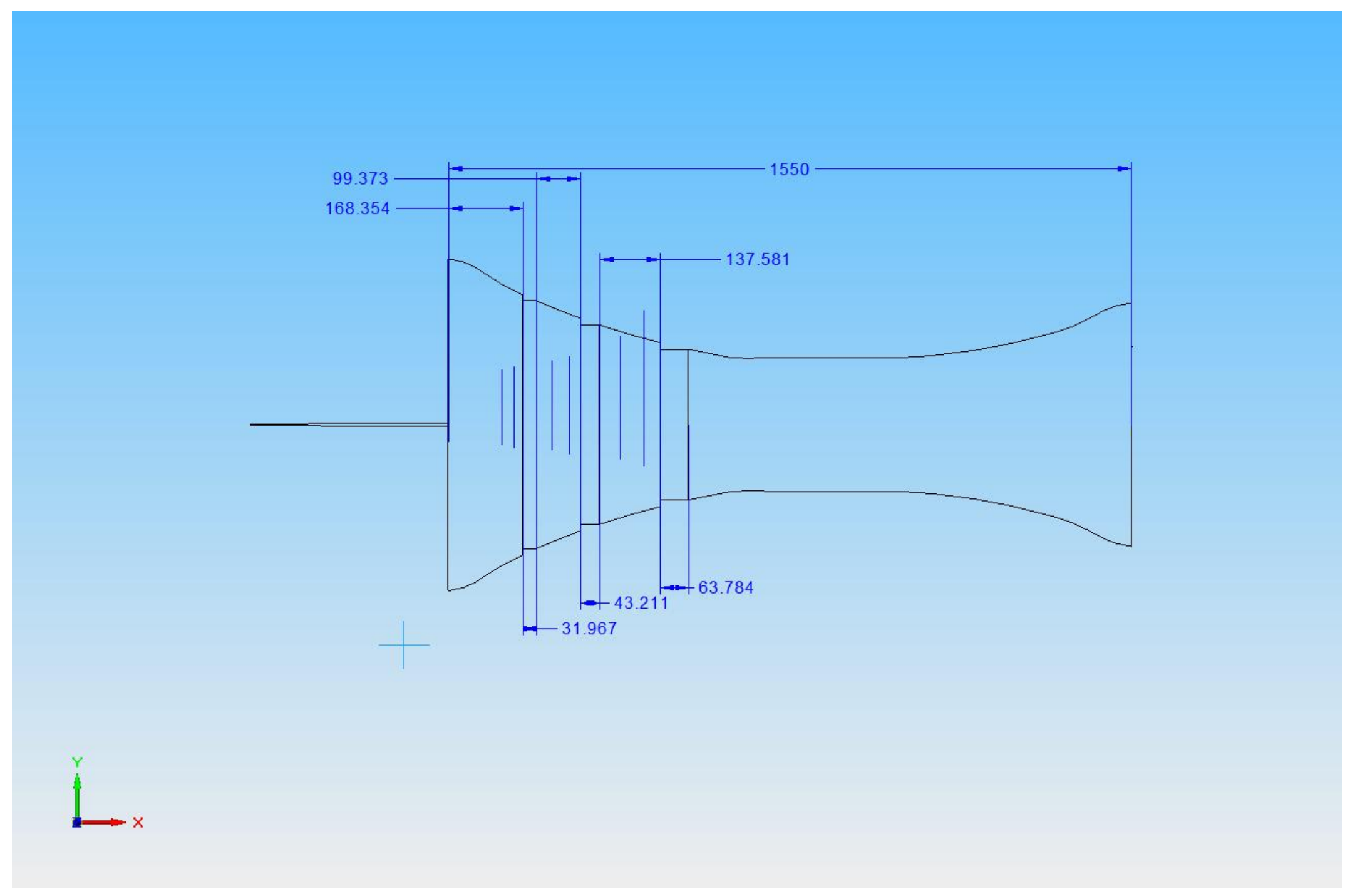

FIGURE 35. Side View (All Units in mm) 


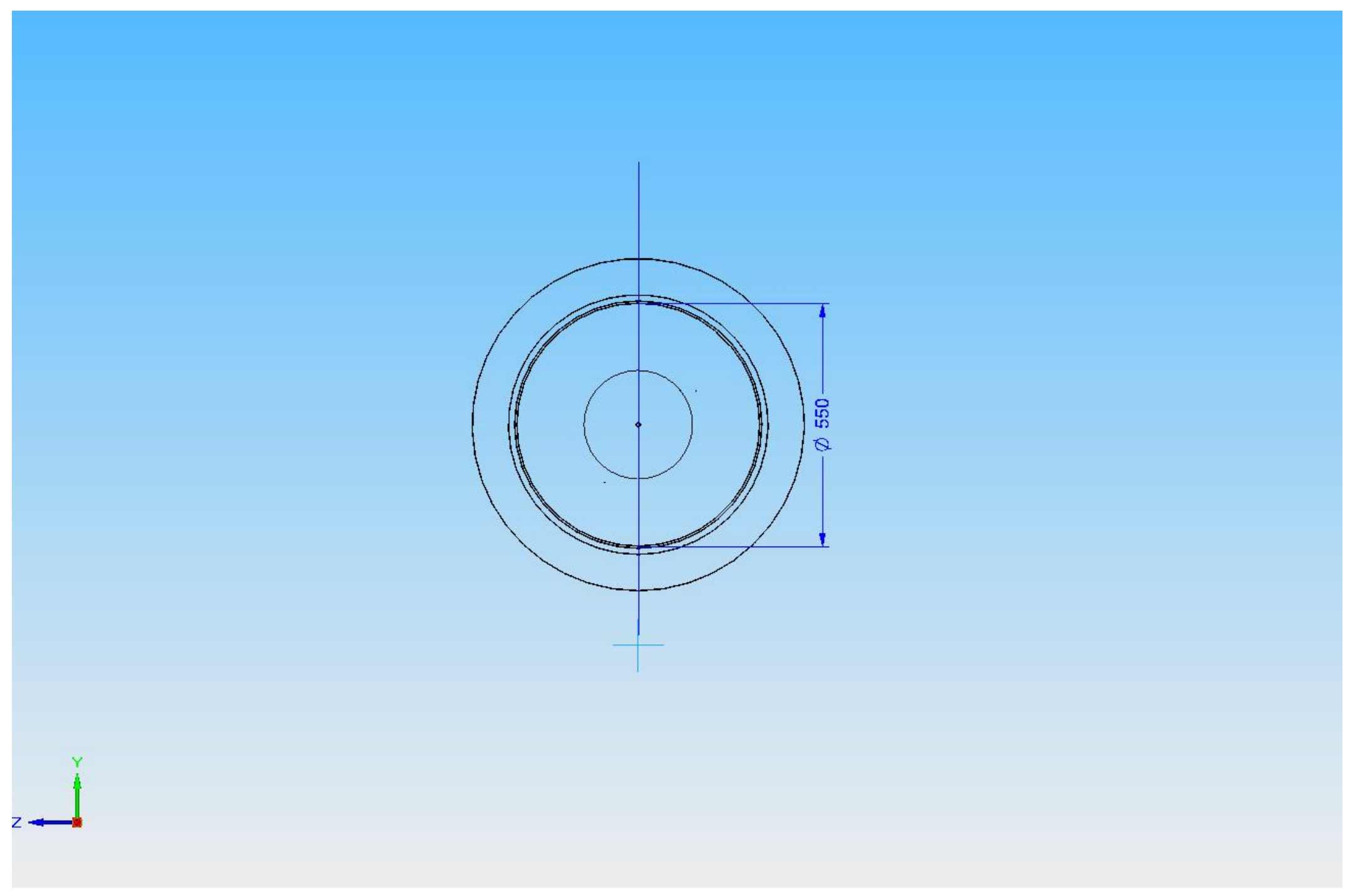

FIGURE 36. Rear View (All Units in mm) 


\section{CURRICULUM VITAE}

Michael Lucas was born in Beckley, West Virginia in 1988. He has lived most of his life in Russell, Kentucky, where he graduated in 2006 from Russell Independent High School. In 2010, he received a B.S. in Chemical Engineering, and expects to graduate in 2011 with a M.S. in Chemical Engineering. His research focuses on the use of computational fluid dynamics to optimize wind power generators. After graduation, he intends to persue other opportunites in the Energy Sector. 